\title{
A Computational and Experimental Study of Flexible Flapping Wing Aerodynamics
}

\author{
Hikaru Aono $^{1 *}$, Satish Kumar Chimakurthi ${ }^{2}$, Pin $\mathrm{Wu}^{3 * *}$, Erik Sällström ${ }^{4 * *}$, Bret K. Stanford ${ }^{5 * *}$, \\ Carlos E. S. Cesnik ${ }^{6}$, Peter Ifju ${ }^{7} * *$, Lawrence Ukeiley ${ }^{8} * *$, Wei Shyy ${ }^{9} *$ \\ *University of Michigan, Ann Arbor, MI, 48109, U. S. A. \\ ** University of Florida, Florida, FL, 32611, U. S. A.
}

To gain some more understanding of the flapping wing aerodynamics and aeroelasticity associated with biological flyers and micro air vehicles (MAVs), a combined computational and experimental study of a well characterized flapping wing structure was conducted. In particular, the coupling between aerodynamics and structural dynamics plays an important role in such flyers but to date has not been adequately addressed. An aeroelasticity framework based on a co-rotational shell finite element solver with a Navier-Stokes solver is developed. Experimentally, a customized digital image correlation system measures the wing deformation, a load sensor attached to the flapping mechanism records the forces produced by the flapping motion, and a stereo digital particle image velocimetry measures the flow velocities. Computational efforts with insight into the fluid physics are reported. Relevant fluid physics are documented including the counter-rotating vortices at the leading and the trailing edge which interact with the tip vortex during the wing motion. Overall, good correlations between experiment and computation are attained. Furthermore, studies on hypothetical flexible flapping wing configurations showed that wing flexibility can be tailored to alter the aerodynamics of a flapping wing.

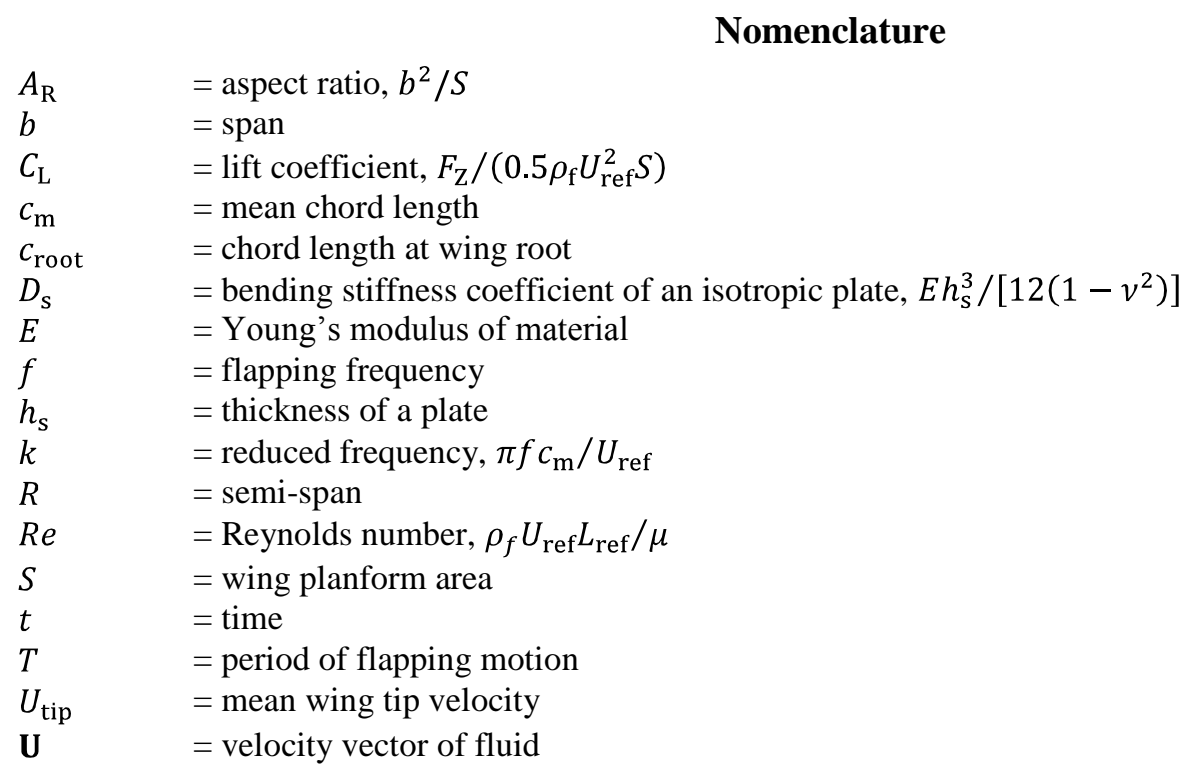

\footnotetext{
${ }^{1}$ Postdoctoral Research Fellow, Aerospace Engineering, aonoh@umich.edu, Member AIAA.

${ }^{2}$ Postdoctoral Research Fellow, Aerospace Engineering, satishkc@umich.edu, Member AIAA.

${ }^{3}$ Graduate Student, Mechanical and Aerospace Engineering, diccidwp@ufl.edu, Member AIAA.

${ }^{4}$ Graduate Student, Mechanical and Aerospace Engineering, eriksallstrom@ gmail.com, Member AIAA.

${ }^{5}$ Currently National Research Council Postdoctoral Fellow, AFRL, bstan@ ufl.edu, Member AIAA.

${ }^{6}$ Professor, Aerospace Engineering, cesnik@ umich.edu, Associate Fellow AIAA.

${ }^{7}$ Professor, Mechanical and Aerospace Engineering, ifju@ufl.edu.

${ }^{8}$ Assistant Professor, Mechanical and Aerospace Engineering, ukeiley@ufl.edu, Associate Fellow AIAA

${ }^{9}$ Clarence L. "Kelly” Johnson Collegiate Professor and Chair, weishyy@umich.edu, Fellow AIAA. 


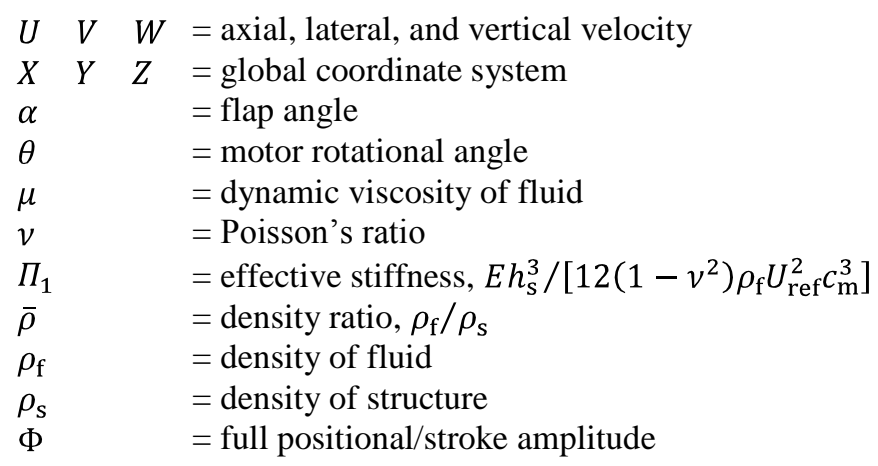

\section{Introduction}

$\mathrm{M}$ icro Air Vehicles (MAVs) have the potential to revolutionize our sensing and information gathering capabilities in areas such as environmental monitoring and homeland security. Numerous vehicle concepts, including fixed wing, rotary wing, and flapping wing, have been proposed ${ }^{1-8}$. As the size of a vehicle becomes smaller than a few centimeters, fixed wing designs encounter fundamental challenges in lift generation and flight control. There are merits and challenges associated with rotary and flapping wing designs. Fundamentally, due to the Reynolds number effect, the aerodynamic characteristics such as the lift, drag and thrust of a flight vehicle change considerably between MAVs and conventional manned air vehicles ${ }^{1-8}$. And, since MAVs are of light weight and fly at low speeds, they are sensitive to wind gusts ${ }^{1-9}$. Furthermore, their wing structures are often flexible and tend to deform during flight. Consequently, the fluid and structural dynamics of these flyers are closely linked to each other. Because of the common characteristics shared by MAVs and biological flyers, the aerospace and biological science communities are now actively communicating and collaborating. Much can be shared between researchers with different training and background including biological insight, mathematical models, physical interpretation, experimental techniques, and design concepts.

Research in the aeroelasticity of flapping wings has substantially increased recently though a full understanding is still lacking. For example, investigations by Daniel and Combes ${ }^{10}$ suggested that aerodynamic loads are relatively unimportant in determining the bending patterns in oscillating wings. Subsequently, experimental investigations by Combes $^{11}$ and Combes and Daniel ${ }^{12}$ found that the overall bending patterns of a Hawkmoth wing are quite similar when flapped (single degree-of-freedom flap rotation) in air and helium, despite a 85\% reduction in fluid density in the latter, suggesting that the contribution of aerodynamic forces is relatively minor compared to the contribution of inertial-elastic forces due to flapping motion. However, they mentioned that realistic wing kinematics might include rapid rotation at the stroke reversal that may lead to increased aerodynamic forces due to unsteady aerodynamic mechanisms ${ }^{1}$. Also, static bending tests by Combes and Daniel ${ }^{11,12}$ showed anisotropy of wing structures in a variety of insect species. More recently, Mountcastle and Daniel ${ }^{13}$ investigated the influence of wing compliance on the mean advective flows (indicative of induced flow velocity) using PIV techniques. Their results demonstrated that flexible wings yield mean advective flows with substantially greater magnitudes and orientations more beneficial to lift than those of stiff wings.

$\mathrm{Zhu}^{14}$ performed a fully coupled fluid-structure interaction analyses to investigate a chordwise flexible airfoil prescribed with pure plunge motion. To clarify the role of inertia on the deformation, the wing was studied in both water and air. Results showed that when the wing is immersed in air, the chordwise flexibility reduces both the thrust and the propulsion efficiency. However, when the wing is immersed in water, it increases the efficiency. Further, he performed numerical investigations on a thin flapping foil prescribed with pure plunge motion in forward flight in different both air and water. The results showed that when the wing was immersed in air, the spanwise flexibility (through equivalent plunge and pitch flexibility) increased the thrust without an efficiency reduction and when the same wing was immersed in water, the spanwise flexibility reduced both the thrust and efficiency. Heathcote and Gursul ${ }^{15}$, Yamamoto et al. ${ }^{16}$, Prempraneerach et al. ${ }^{17}$, Tang et al..$^{18}$, and Chandar and Domadran ${ }^{19}$ presented studies on both rigid and partially chordwise flexible airfoils prescribed with both pure plunge and/or combined plunge/pitch motions in water and showed that flexible wings may be more efficient than the rigid ones. In particular, Pederzani and Haj-Hariri ${ }^{20}$ suggested that lighter plunging airfoils are capable of generating more thrust than heavier ones and are more efficient. They performed computational analyses on a rigid wing from which a portion was cut out and covered with a very thin and flexible material (latex) and showed that due to a snapping motion (i.e. non zero velocity in the direction opposite to that of the following stroke) of the latex at the beginning of 
each stroke, the strength of the vortices that are shed is higher in lighter wing structures, leading to the generation of more thrust. Furthermore, such structures require less input power in order to be snapped than heavier ones. Chaithanya and Venkatraman ${ }^{21,22}$ investigated the influence of inertial effects due to prescribed motion on the thrust coefficient and propulsive efficiency of a plunging/pitching thin plate using inviscid flow theory and beam equations. Their results demonstrate that flexible airfoils with inertial effects yield more thrust than those without inertial effects. This is due to the increase in the fluid loading in the former which subsequently lead to an increase in the deformation. Due to their shape, deformed airfoils produce a force component along the forward velocity direction $^{22}$. Gopalakrishnan ${ }^{23}$ analyzed the effects of elastic cambering of a rectangular membrane flapping wing on aerodynamics in the forward flight using a linear elastic membrane solver coupled with an unsteady LES method. Different membrane pre-stresses were investigated to give a desired camber in response to the aerodynamic loading. The results showed that the camber introduced by the wing flexibility increased the thrust and lift production considerably. Analysis of flow structures revealed that the LEV stayed attached on the top surface of the wing, followed the camber, and covered a major part of the wing, which resulted in high force production. On the other hand, for rigid wings (which were also considered) the leading edge vortex lifted off from the surface resulting in low force production.

Miao and $\mathrm{Ho}^{24}$ prescribed a time-dependent flexible deformation profile to an airfoil in pure plunge and investigated the effect of flexure amplitude on the unsteady aerodynamic characteristics for various combinations of Reynolds number and reduced frequency. For a specific combination of Reynolds number, reduced frequency, and plunge amplitude, the results showed that thrust-indicative wake structures are observed behind the trailing edge of the airfoil for airfoils with flexure amplitudes of $0.0-0.5$ of the chord length. It was shown that, this wake structure evolved into a drag-indicative form as the flexure amplitude of the airfoil was increased to 0.6 and 0.7 of the chord length. Studies conducted under various combinations of Reynolds number and reduced frequency showed that the propulsive efficiency of a chordwise flexible airfoil in pure plunge is influenced primarily by the value of the reduced frequency rather than by that of the Reynolds number. Toomey and Eldredge ${ }^{25}$ performed numerical and experimental investigations to understand the role of flexibility in flapping wing flight using two rigid elliptical sections connected by a hinge with torsion spring. The section at the leading edge was prescribed with fruit fly-like hovering wing kinematics ${ }^{1}$, while the trailing edge section responded passively due to the fluid dynamic and inertial/elastic forces. It was found that the lift force and wing deflection are primarily controlled by the nature of the wing rotation. Faster wing rotation, for example, led to larger peak deflection and lift generation. Advanced rotation also led to a shift in the instant of peak wing deflection which increased the mean lift. In contrast to the rotational kinematics, the translational kinematics were shown to have very little impact on spring deflection or force. And, while the former was shown to be nearly independent of Reynolds number, the latter was shown to increase with increasing Reynolds number.

Vanella et $a l .{ }^{26}$ conducted numerical investigations on a similar structure and found that approximately $30 \%$ increase in lift is realized when the wing is excited by a non-linear resonance at $1 / 3^{\text {rd }}$ of its natural frequency. For all Reynolds numbers considered, the wake capture mechanism was enhanced due to a stronger flow around the wing at stroke reversal, resulting from a stronger vortex at the trailing edge. Heathcote et al..$^{27}$ investigated the effect of chordwise flexibility on aerodynamic performance of an airfoil in pure plunge under hovering conditions. Because the trailing edge is a major source of shedding of vorticity at zero freestream velocity, they showed that the amplitude and phase angle of the motion of the trailing edge affect the strength and spacing of the vortices, and the time averaged velocity of the induced jet. Direct force measurements confirmed that at high plunge frequencies, the thrust coefficient of the airfoil with intermediate stiffness is highest, although the least stiff airfoil can generate larger thrust at low frequencies. It was suggested that there is an optimum airfoil stiffness for a given plunge frequency and amplitude. Similar conclusions were made in another study ${ }^{28}$ wherein, the influence of resonance on the performance of a chordwise flexible airfoil prescribed with pure plunge motion at its leading edge was studied. It was shown that while the mean thrust could increase with an increase in flexibility, above a certain threshold the wing is too flexible to communicate momentum to the flow. Further, too much flexibility led to a net drag and hence only a suitable amount of flexibility was desirable for thrust generation.

Numerical simulations were performed by Liu and $B_{0 s}{ }^{29}$ for a 3-D pitching and plunging wing in forward flight. Their results showed that the phase of the flexing motion of the wing relative to the prescribed heave motion plays a key role in determining thrust and efficiency characteristics of the fin. Heathcote et al. ${ }^{30}$ conducted water-tunnel studies to study the effect of spanwise flexibility on the thrust, lift, and propulsive efficiency of a plunging flexible wing configuration in forward flight.

Hamamoto $\mathrm{et} \mathrm{al}^{31}$ conducted a fluid-structure interaction analysis on a deformable dragonfly wing in hover and examined the advantages and disadvantages of flexibility. They tested three types of flapping flight: a flexible wing driven by dragonfly flapping motion, a rigid wing (stiffened version of the original flexible dragonfly wing) driven 
by dragonfly flapping motion, and a rigid wing driven by modified flapping based on tip motion of the flexible wing. They found that the flexible wing with nearly the same average energy consumption generated almost the same amount of lift force as the rigid wing with modified flapping motion, which realized the same angle of attack at the aerodynamically dominant sections of the wing. However, the rigid wing required $19 \%$ more peak torque and $34 \%$ more peak power, indicating the usefulness of wing flexibility. Singh and Chopra ${ }^{32}$ developed an experimental apparatus with a bio-inspired flapping mechanism to measure the thrust generated for a number of wing designs. The key conclusions that stemmed from this study were that the inertial loads constituted the major portion of the total loads acting on the flapping wings tested on the mechanism and that for all the wings tested, the thrust dropped at higher frequencies. Further, the author found that at such frequencies, the light-weight and highly flexible wings used in the study exhibited significant aeroelastic effects. Young et al. ${ }^{33}$ conducted numerical investigations on a tethered desert locust called Schistocerca gregaria ${ }^{34}$. Results demonstrated that time-varying wing twist and camber were essential for the maintenance of attached flow. The authors emphasized that while high-lift aerodynamics was typically associated with massive flow separation and large LEVs, when high lift was not required, attached flow aerodynamics could offer greater efficiency. Their results further showed that, in designing robust lightweight wings that could support efficient attached flow, it was important to build a wing that undergoes appropriate aeroelastic wing deformation through the course of a wing beat.

Agrawal and Agrawal ${ }^{35}$ investigated the benefits of insect wing flexibility on flapping wing aerodynamics based on experiments and numerical simulations. They compared the performance of two synthetic wings: (i) a flexible wing based on a bio-inspired design of the hawkmoth (Manduca sexta) wing and (ii) a rigid wing of similar geometry. The results demonstrated that more thrust was generated by the bio-inspired flexible wing compared to the rigid wing in all wing kinematic patterns considered. They emphasized that the results provided motivation for exploring the advantages of passive deformation through wing flexibility and that coupled fluid-structure simulations of flexible flapping wings were required to gain a fundamental understanding of the physics and to guide optimal flapping wing MAV designs.

Wu et $a l .{ }^{36}$ and Sällström et al. ${ }^{37}$ presented a multidisciplinary experimental endeavor correlating flapping wing MAVs aeroelasticity and thrust production by quantifying and comparing elasticity, dynamic responses, and air flow patterns of six different pairs of MAV wings (in each one, the membrane skin was reinforced with different leading edge and batten configurations) of the Zimmerman planform (two ellipses meeting at the quarter chord) with varying elastic properties. In their experiment, single degree-of-freedom flapping motion was prescribed to the wings in both air and vacuum. Amongst many conclusions, they found that, within the range of flexibility considered, more flexible wings are more thrust-effective at lower frequencies whereas stiffer wings are more effective at higher frequencies. They hypothesized that flexible wings may have a certain actuation frequency for peak thrust production and the performance would degrade once that frequency is passed.

These studies as well as the investigations of Heathcote et $a l . .^{27,30}$, Chimakurthi et $a l .^{38}$, Aono et al. ${ }^{39}$, and Tang et $a l^{18}$ offer consistent findings. It seems like the spanwise flexibility increases aerodynamic forces by creating higher effective angles of attack via spanwise deformation. However, apart from affecting the overall aerodynamic force generation, the chordwise flexibility can redistribute lift versus thrust by changing the projection angle of the wing with respect to the freestream by changing airfoil via camber deformation, for example. Of course, similar behavior may be observed in spanwise flexible with passive twist deformation along the wing span. Overall, both spanwise and chordwise flexibility need to be considered together in order to optimize the aerodynamic performance under different flight speed and environment uncertainty such as wing gust.

In order to analyze plate/shell-like structures flapping in incompressible, viscous flow within the low Reynolds number regime, a newly developed aeroelasticity framework ${ }^{40}$ is used. It is based on a partitioned solution of flow and structural solvers capable of supporting implicit coupling ${ }^{38,39}$. The key features of the present aeroelasticity framework are discussed in the next section. The computational analyses are assessed with experimental results under clearly characterized conditions. Specifically, we highlight our ongoing efforts geared towards developing an integrated computational and experimental approach to perform aeroelastic analyses of flapping wings within various configurations. Experimentally, a customized digital image correlation system measures the wing deformation, a load sensor attached to the flapping mechanism records the forces produced by the flapping motion, and a stereo digital particle image velocimetry captures the flow structure around the wings. Detailed comparisons between experimental and computational efforts with insight into the fluid physics are reported. Furthermore, the impact of wing flexibility on flapping wing aerodynamics is presented. 


\section{Numerical Framework for High-Fidelity Flapping Wing Simulations}

In this section, a brief description of the fluid and structural dynamics approaches for the aeroelastic analysis of flapping wing is presented. From these, an aeroelastic framework is developed for the analysis of low Re flows and their interactions with flexible flapping wings.

\section{A. Computational Fluid Dynamics Modeling (STREAM)}

The governing equations of fluid are the unsteady, incompressible 3-D Navier-Stokes equations and the continuity equation, which are expressed in vector form as follows:

$$
\begin{gathered}
\frac{\partial \mathbf{U}}{\partial t}+\mathbf{U} \cdot \nabla \mathbf{U}=-\frac{1}{\rho_{f}} \nabla p+\frac{\mu}{\rho_{f}} \nabla^{2} \mathbf{U}, \\
\nabla \cdot \mathbf{U}=0,
\end{gathered}
$$

where $\rho_{\mathrm{f}}$ is the fluid density, $\mu$ is the dynamic viscosity coefficient, $\mathbf{U}=\left(\begin{array}{lll}U & V & W\end{array}\right)$ is the velocity vector of the fluid, $t$ is the time, $\mathbf{X}=\left(\begin{array}{llll}X & Y & Z\end{array}\right)$ is the position vector of the fluid based on the inertial frame, $\nabla$ is the gradient operator with respect to $\mathbf{X}$, and $p$ is the pressure, respectively.

The flapping wing velocity is chosen as the velocity scale, then the resulting non-dimensional form of the NS equations is:

$$
\frac{k}{\pi} \frac{\partial \overline{\mathbf{U}}}{\partial \bar{t}}+\overline{\mathbf{U}} \cdot \bar{\nabla} \overline{\mathbf{U}}=-\bar{\nabla} \bar{p}+\frac{1}{R e} \bar{\nabla}^{2} \overline{\mathbf{U}}
$$

where the over-bar designates the dimensionless variable. This form of the equation separates the reduced frequency $\left(k=\pi f c_{\mathrm{m}} / U_{\text {ref }}\right)$ and the Reynolds number $\left(R e=\left(\rho_{\mathrm{f}} L_{\mathrm{ref}} U_{\mathrm{ref}}\right) / \mu\right)$, making it convenient to study the effects of these parameters.

The numerical solution is obtained using a pressure-based algorithm, with an employment of combined Cartesian and contravariant velocity variables to facilitate strong conservation law formulations and consistent finite-volume treatment. The convection terms are discretized using a second-order upwind scheme, while the pressure and viscous terms with a second-order central difference scheme. For the time integration, an implicit Euler scheme is employed. A moving grid technique employing the master-slave concept ${ }^{1,7}$ is used to re-mesh the multi-block structured grid for fluid-structure interaction problems. The geometric conservation law (GCL) originally proposed by Thomas and Lombard $^{41}$ was incorporated to compute the cell volumes in the moving boundary problem consistently and eliminate artificial mass sources. The specific implementation and implications of the GCL in the context of the present solution algorithm have been discussed by Shyy et al. ${ }^{42}$.

\section{B. Structural Dynamics Solution (UM/NLAMS)}

The nonlinear structural dynamics solution is based on a flexible multi-body type finite element analysis of a flapping wing using triangular shell elements. It relies on the use of a body-fixed floating frame of reference to describe the prescribed rigid body motion and on a co-rotational (CR) framework to account for geometric nonlinearities. The solution is implemented in the "University of Michigan's Nonlinear Membrane Shell Solver" (UM/NLAMS), written in Fortran 90. The CR formulation has generated a great amount of interest in the last decade. The idea of this approach is to decompose the motion into rigid body and pure deformational parts through the use of a local frame at each finite element which translates and rotates with the element. The element's internal force components are first calculated relative to the co-rotational frame and are then transformed to a global frame using the co-rotational transformation matrix. The co-rotational frame transformation eliminates the element rigid body motion so that a linear deformation theory can be used. Hence, the main advantage of the CR formulation is its effectiveness for problems with small strains but large rotations. The elastic stiffness matrix for the shell element in this work will be obtained as a superposition of the stiffnesses corresponding to that of the optimal membrane element (OPT) and a discrete Kirchhoff triangle (DKT) plate bending element. Full details of the structural dynamics solution are available in Chimakurthi et al. ${ }^{40}$

\section{Aeroelastic Coupling}

The aeroelastic coupled solution is based on a time-domain partitioned solution process in which the nonlinear partial differential equations modeling the dynamic behavior of both fluid and structure are solved independently with boundary information (aerodynamic loads and structural displacements) being shared between each other alternately. A dedicated interface module was developed to enable communication between the flow and the structure at the 3-D wetted surface (fluid-structure interface). In the interface module, both the fluid and the 
structural modules are called one after the other according to the coupling method adopted for the problem. The coupling algorithm is determined by the capability of the individual simulation code. More details about the coupling aspects can be found in Chimakurthi et al. ${ }^{38,40}$ and Aono et al. ${ }^{39}$

\section{Experimental Setup for Flapping Wing Experiments}

\section{A. Flapping Mechanism and Tested Wings}

A single-degree-of-freedom flapping mechanism is designed and built for this study, as shown in Figure 1 (the same mechanism as the one used in reference ${ }^{35}$, but the wings shown in the figure are different from the aluminum wings tested). The design is created based on a Maxon motor system that includes a $15 \mathrm{~W}$ brushless DC motor EC16, a 57/13 reduction ratio planetary gear head, a 256 counts-per-turn encoder and an EPOS 24 controller. This system provides precise control of the motor system: the sensor provides position and velocity feedback to the controller that actively regulates the motor. Utilizing the high precision pre-assembled planetary gear head rather than constructing a custom gear transmission is also advantageous. The final output range of the motor shaft is: speed 0 to 45 revolutions per second (RPS) and nominal torque 0 to $21 \mathrm{Nmm}$.

The rotation output from the motor is first transformed into a reciprocating motion with a crank-slider mechanism; then a bar linkage mechanism realizes the flapping motion at the wing mount. A detailed schematic description of the flapping kinematics is presented in Figure 1. The geometric relationship between motor rotation (angle $\theta$ ) and flap angle $\alpha$ is expressed in the equations in the figure, where $\alpha$ is the flap angle; $\theta$ is the motor rotational angle; $x$ is the vertical displacement from the center point when the wings are horizontally positioned. The rest of the parameters are selected so that $\mathrm{a} \pm 21^{\circ}$ amplitude is maintained. The experiments are performed at $10 \mathrm{~Hz}$ flapping frequency.

The wing planform is of a 7.65 aspect ratio and has a Zimmerman shape, i.e. is formed by two ellipses which intersect at the quarter-chord point. The wing length is $75 \mathrm{~mm}$ and the root chord length is $25 \mathrm{~mm}$. The wings are manufactured with CNC machine to achieve the exact contour and avoid stresses that may cause warping. After machining, the wing surface is primed with flat white coating for the background and speckled with black dots for DIC measurements. Another coating (Rhodamine) is applied for reducing blooming of the laser sheet hitting the wing during PIV measurements. The final weight of a single wing is $1.685 \pm 0.005$ grams. The flatness is examined with DIC and bounded within $-0.05 \sim 0.05 \mathrm{~mm}$. The wing is mounted to the mechanism at a $5 \times 5 \mathrm{~mm}^{2}$ square region at the crossing of the leading edge and root. This region of the wing is assumed not to deform during the flapping motion (therefore serving as the reference for calculating the wing deformation and also the boundary condition).

\section{B. Deformation Measurement with DIC}

The kinematics and deformation of the flapping wings are measured with a high speed digital image correlation system (see Figure 2). DIC is a well-developed non-contact measurement technique used to capture full-field displacement and deformation of surfaces via stereo-triangulation. A random speckle pattern is applied to the flapping wing, which is then digitized into wing surface coordinates with stereo triangulation. The full-field displacements of the wing during the flapping motion are computed with temporal matching, by minimizing a cross correlation function between discrete regions of speckle patterns on a deformed wing surface and an undeformed one. The DIC system (Correlated Solutions Inc., South Carolina, USA) used in this study consists of two Phantom high speed cameras that can store 2800 pictures of 800 pixels by 600 pixels resolution in its flash memory at a maximum rate of 4800 frames per second. The exposure time was $150 \mu \mathrm{s}$ and the frame rate was set to obtain 100 frames per cycle. For example, in the $10 \mathrm{~Hz}$ case therefore, the frame rate is $1000 \mathrm{fps}$. 


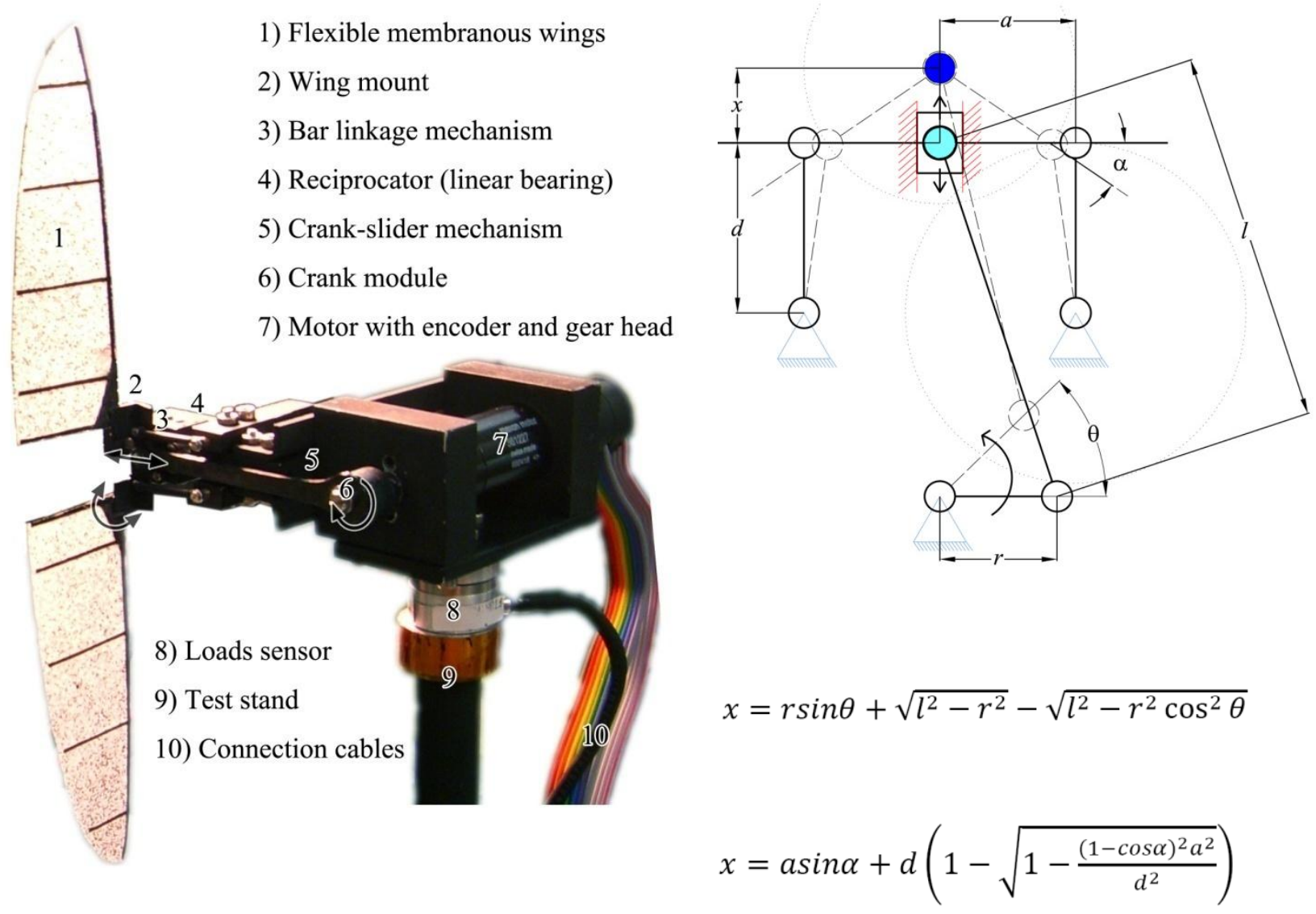

Figure 1. Flapping mechanism and its schematics.
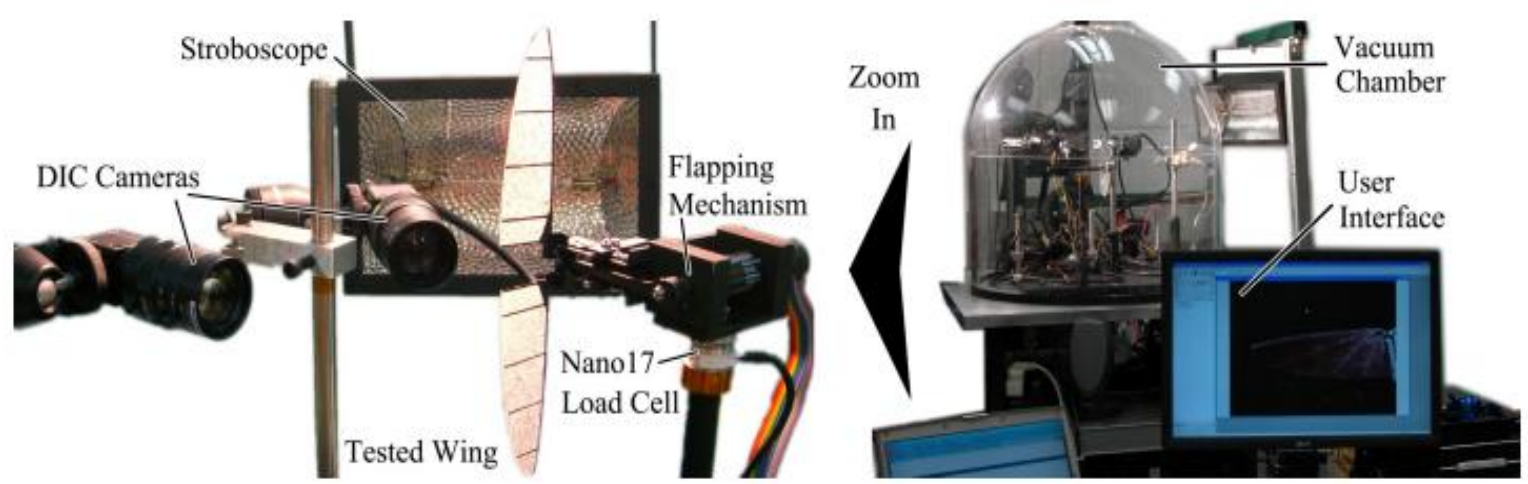

Figure 2. Digital image correlation (DIC) experimental setup

Only the left wing is measured with DIC for the kinematics and deformation. The two DIC cameras are symmetrically placed facing the wings at mid-plane. Field of view on both cameras is adjusted to fit and fill the frame. Then the cameras are calibrated and used to measure the full-field deformation and kinematics of the flapping wing. A reference image at the mid-plane of stroke is taken beforehand. The data structure contains coordinates of all points on the measurement surface. During post processing, the structural deformation is separated from rigid body kinematics by comparing the undeformed reference data to the deformed wing at the same flap angle. The errors of DIC are induced during the calibration phase and the uncertainty will remain the same after calibration. If a 
camera pair is carefully calibrated, the system uncertainty is under $\pm 0.1 \mathrm{~mm}$ in the current setup (except occasionally the speckle pattern introduces other error).

\section{Airflow Measurement with Particle Image Velocimetry}

Particle image velocimetry, a mature technique used to measure the velocity of particles (assumed to follow the airflow) in the flow of interest ${ }^{1,13}$, is applied to examine the airflow around a flapping wing. The flow field measurement area is defined by the confluence of the position and dimension of a laser sheet, along with the camera's field of view. Images are captured at each laser flash so that the particle spatial displacement can be extracted by correlating two images taken within a small time interval. One LaVision Imager pro X $4 \mathrm{M}$ camera capable of capturing up to 7 image pairs per second with a pixel resolution of $2048 \times 2048$ is used. The camera is mounted perpendicular to the wing root, measuring a plane in the wing span direction at $7 \mathrm{~Hz}$ frame rate. A laser sheet is generated from a Litron Nano L PIV Pulsed Nd:YAG Laser System (max energy $135 \mathrm{~mJ} / \mathrm{pulse}, 532 \mathrm{~nm}$ ) and is directed at the target from below. The air is seeded by a LaVision Aerosol Generator, with olive oil, an modal radius of approximately 0.25 microns. Davis 7 (software from LaVision) is used to control the PIV system and process the data. The measurements are made in the test section of a sealed open jet wind tunnel. Snapshots of the velocity field are acquired at 3 different chord locations $(0.25,0.5$ and 0.75 of the chord length at the root).. The measurement area is $121 \times 121 \mathrm{~mm}^{2}$, enclosing the wing at the upper right corner.

The PIV images are captured at up to $7 \mathrm{~Hz}$, with the snapshots skipped that the system does not have time to process. 2500 images are captured at each location and flapping frequency. The images are processed using a multipass algorithm with shrinking interrogation size to produce velocity vectors. The region next to the wing and the area where the laser is shaded by the wing are masked out. The raw images are then processed to find the wing angle. The wing angle is then fit to a sine wave to extract the phase of the wing in each snapshot. The snapshots are then divided into 50 bins, with the bins equally spaced in time within the flapping cycle. Phase averages are then produced by averaging each bin. The wing angle variation within a bin varies between approximately $\pm 0.5^{\circ}$ to $\pm 2.5^{\circ}$, and each bin contains on the average 50 snapshots.

\section{Results and Discussions}

Preliminary tests have been conducted on an elliptic isotropic wing using the computational aeroelasticity framework involving UM/NLAMS and STREAM and the experimental setup described above. A brief description of the test along with results is presented below:

\section{Aeroelastic response for a Zimmerman aluminum wing prescribed with pure single DOF flap rotation}

The aeroelastic response was computed for an isotropic Zimmerman aluminum wing flapping in incompressible, viscous flow under hovering conditions at $10 \mathrm{~Hz}$. A summary of the geometric and mechanical properties of the aluminum wing configuration studied in the experiment and computations is included in Table 1. Table 2 provides information about the flow properties (dimensional). In Table 3, the key dimensionless parameters related to either the structure, the flow, or to both for the aluminum wing configuration are furnished. The dimensionless parameters $\bar{\rho}$ and $\Pi_{1}$ are defined in Shyy et al. ${ }^{1}$. An O-type structured multi-block grid around the Zimmerman wing of aspect ratio 7.65 is used for the computational fluid dynamics (CFD) simulations. The finite element mesh configuration developed in UM/NLAMS has triangular shell finite elements. A $5 \mathrm{~mm} \times 5 \mathrm{~mm}$ square region near the root at the leading edge is constrained in all degrees of freedom (with respect to the global frame) in the structural solver, since the flapping mechanism in the experiment is used to actuate that region on the wing. A total of 480 elements (275 nodes) are used in the finite element discretization.

To assess the independence of the numerical solution to CFD grid refinement, a grid convergence study was performed and a suitable grid (approximately total 0.7 million cells) was subsequently chosen. A time step of 1.5 $\times 10^{-3} \mathrm{~s}$ (and hence approximately 650 time-steps per period of computation) was used for the computations. The convergence criterion for the aeroelastic convergence was chosen as a check on the Euclidean norm of the entire solution vector computed in two consecutive fluid-structure sub-iterations. 
Table 1. Geometric and mechanical properties associated with the test case.

\begin{tabular}{lcl}
\hline \hline Quantity & Symbol & Value \\
\hline \hline Semi-span at quarter chord & $R$ & $0.075 \mathrm{~m}$ \\
Chord length at wing root & $c_{\text {root }}$ & $0.025 \mathrm{~m}$ \\
Structural thickness & $h_{\mathrm{s}}$ & $0.4 \times 10^{-3} \mathrm{~m}$ \\
Poisson's ratio & $v$ & 0.3 \\
Material density & $\rho_{\mathrm{s}}$ & $2700 \mathrm{~kg} / \mathrm{m}^{3}$ \\
Young's modulus of material & $E$ & $70.0 \mathrm{GPa}$ \\
\hline \hline
\end{tabular}

Table 2. Flow properties associated with the test case.

\begin{tabular}{lcc}
\hline \hline Quantity & Symbol & Value \\
\hline \hline Reference flow velocity & $U_{\text {ref }}$ & $1.0995 \mathrm{~m} / \mathrm{s}$ (hover) \\
Air density & $\rho_{\mathrm{f}}$ & $1.209 \mathrm{~kg} / \mathrm{m}^{3}$ \\
\hline \hline
\end{tabular}

Table 3. Dimensionless parameters associated with the test case.

\begin{tabular}{lcl}
\hline Quantity & Symbol & Value \\
\hline \hline Mean chord-based Reynolds number & $R e$ & 2605 \\
Reduced frequency & $k$ & 0.56 \\
Aspect ratio & $A_{\mathrm{R}}$ & 7.65 \\
Density ratio & $\bar{\rho}$ & 2233 \\
Scaling parameter - I & $\Pi_{1}$ & $3.8 \times 10^{4}$ \\
\hline \hline
\end{tabular}

It may be noted that the wing is defined in the $X-Z$ plane wherein, the $X$-axis goes through the wing chord and the $Z$-axis going through the wing length. Figures 3 and 4 show a comparison of the velocity magnitude and vorticity between computation and the experiment for two different points in the flapping cycle $(t / T=0.3$ and 0.48 , where $T$ is the period of prescribed flap rotation) respectively. The flow field in these plots is shown on a slice that is cut at the quarter chord station going through the span. The experimental data could not be obtained in the region near the wing that is outside the laser sheet (appears as a white cone-like region above the wing in the experimental velocity magnitude and vorticity contours).

As seen from the plots, there is a good overall agreement in the flow structure between the computation and the experiment at both points in the flapping cycle. However, the vorticity is weaker and more fragmented in the experiment than in the computation and the magnitude of velocity is larger than the experimental one. Also, in general, there is more discrepancy near the wing tip than in the rest of the wing. This is confirmed from the comparison of the velocity distributions between the computation and the experiment shown in Figure 5. Each of those sub-plots is obtained by considering a line of points vertically above and below the wing obtained by intersecting a slice going through the quarter chord all along the span and another slice at either a section near the mid-span or the tip that goes through the entire chord. For example, the sub-plots (A-1) and (B-1) of Figure 5 show such velocity magnitude distributions corresponding to a line of points obtained by intersecting the chordwise slice at mid-span and tip respectively one after the other with the slice going through the span at the quarter chord, both for the time instant $t / T=0.3$. Then, the sub-plots (C-1) and (D-1) of Figure 5 correspond to the same line of points but now for time instant $t / T=0.48$. 


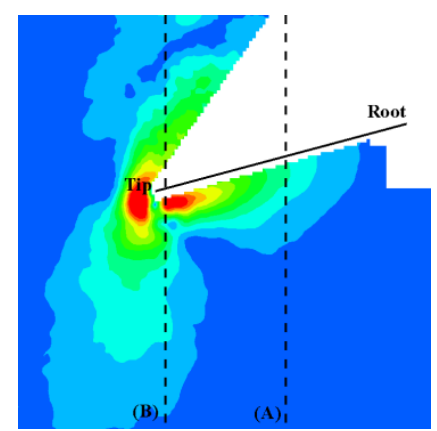

Experiment (Phase averaged)

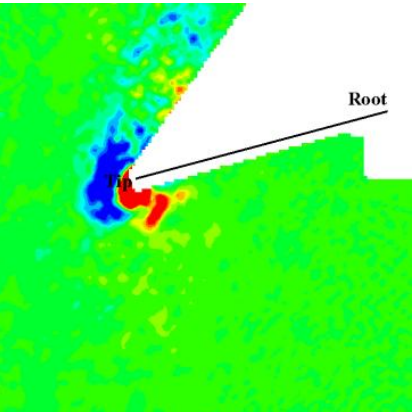

Experiment (Phase averaged)

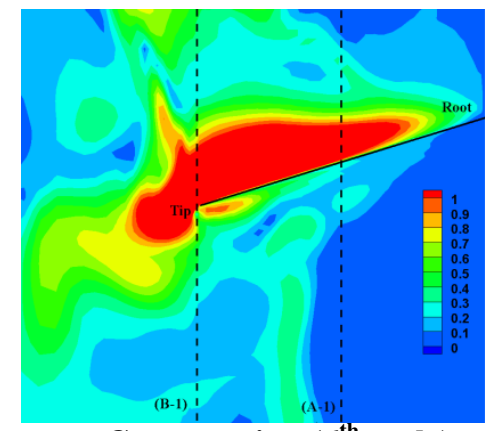

Computation $\left(6^{\text {th }}\right.$ cycle $)$

\section{Velocity magnitude $[\mathrm{m} / \mathrm{s}]$}

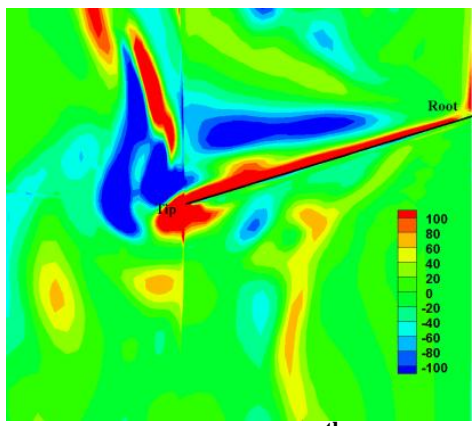

Computation $\left(6^{\text {th }}\right.$ cycle $)$

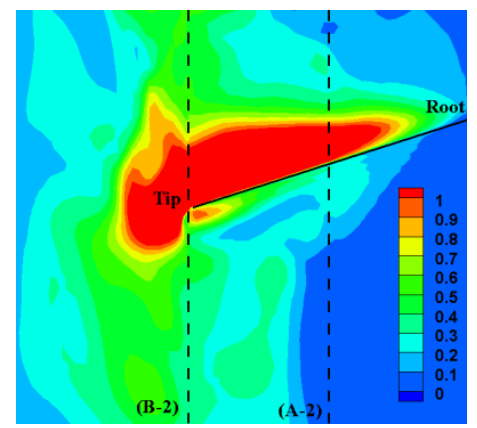

Computation (Phase averaged)

\section{$\underline{X-V o r t i c i t y ~}[1 / s]$}

Figure 3. Comparison of velocity magnitude and vorticity between experiment and computation for a slice of the wing at the quarter chord going through the span for time instant $t / T=0.3$. The sub-plots in the first column correspond to the phase averaged velocity magnitude and $X$-vorticity contours of the experimental data. The sub-plots in the second column correspond to the velocity magnitude and $X$-vorticity contours corresponding to the $6^{\text {th }}$ cycle of computation. The sub-plots in the third column correspond to the phase averaged (using the data corresponding to the $3^{\text {rd }}, 4^{\text {th }}, 5^{\text {th }}$, and $6^{\text {th }}$ cycles) velocity magnitude and $X$-vorticity contours of the computational data. $\left(\operatorname{Re}=2605, k=0.56, \Pi_{1}=3.8 \times 10^{4}, \bar{\rho}=2233\right)$.

Also included in Fig. 5 are phase averaged velocity profiles for both points in the cycle, $t / T=0.3$ and $t / T=0.48$, (sub-plots A-2, B-2 correspond to the former time instant and C-2, D-2 correspond to the latter time instant) including the error bars for the experimental data. While there was good qualitative agreement from the contour plots in Figures 3 and 4, here one can see a more quantitative comparison. In general, there are cycle-to-cycle variations in the velocity magnitudes in both computation and experiment. Notwithstanding that, specifically, it appears that the spatial locations associated with the rise in velocities due to the wing motions do not match too well between the experiments and computations however the maximum amplitudes of the velocity magnitudes do agree quite well. In the experimental data, the magnitudes of variance near the tip region are generally larger than those near the mid-span. Figure 6 shows the lift coefficient computed from numerical data on the wing as a function of non-dimensional time. Figure 7 shows the iso-surfaces of the vorticity magnitude (the color corresponds to the spanwise vorticity) corresponding to two different time instants labeled as (a) and (b) in Figure 6. Three-dimensional vortex generation is seen in Figure 7 at both time instants wherein the counter-rotating vortices at the leading and the trailing edge interact with the tip vortex during the wing motion. In particular, the vortices generated during a previous stroke (indicated as "PV" in the figure) are captured by the wing and interact with the vortices generated during a current stroke indicated as "CV". 


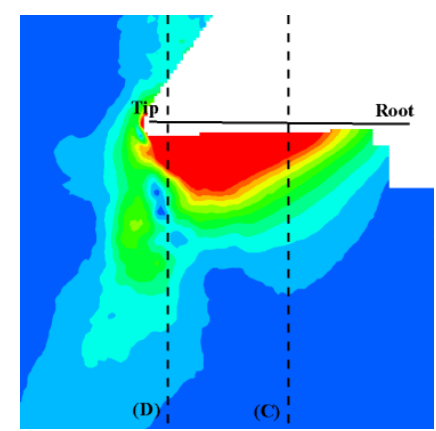

Experiment (Phase averaged)

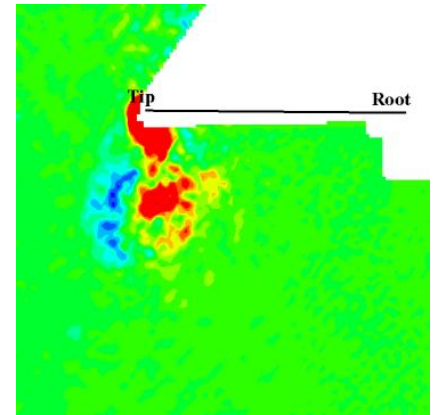

Experiment (Phase averaged)

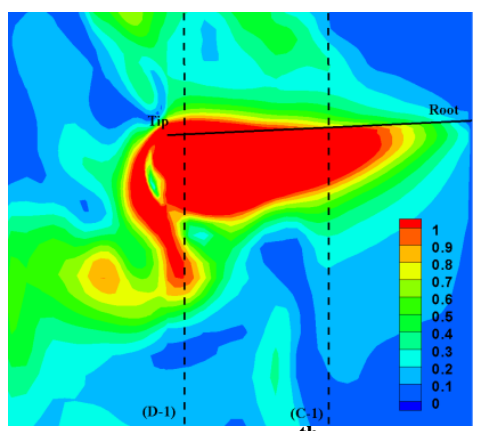

Computation $\left(6^{\text {th }}\right.$ cycle) Velocity magnitude $[\mathrm{m} / \mathrm{s}]$

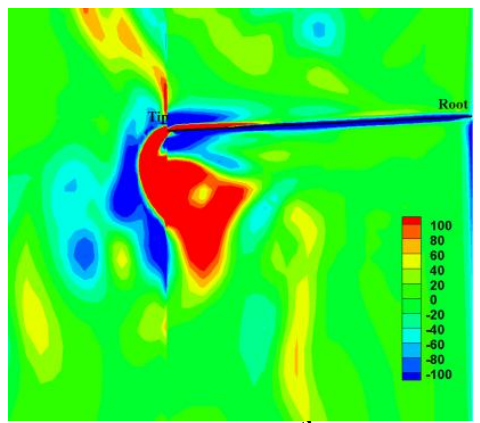

Computation $\left(6^{\text {th }}\right.$ cycle $)$ X-Vorticity $[1 / s]$

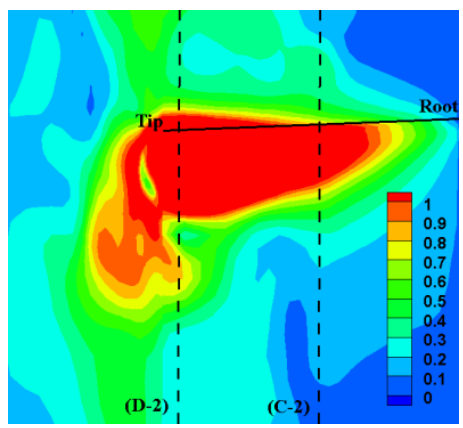

Computation (Phase averaged)

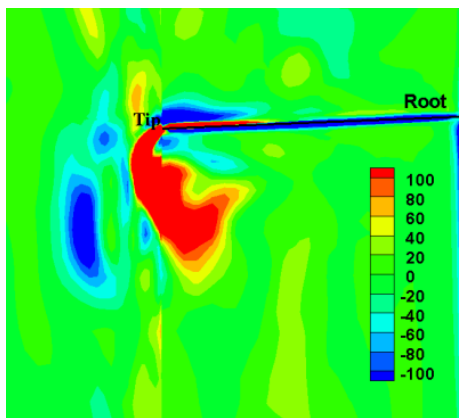

Computation (Phase averaged)

Figure 4. Comparison of velocity magnitude and vorticity between experiment and computation for a slice of the wing at the quarter chord going through the span for time instant $t / T=0.48$. The sub-plots in the first column correspond to the phase averaged velocity magnitude and $X$-vorticity contours of the experimental data. The plots in the second column correspond to the velocity magnitude and $X$-vorticity contours corresponding to the $6^{\text {th }}$ cycle of computation. The plots in the third column correspond to the phase averaged (using the data corresponding to the $3^{\text {rd }}, 4^{\text {th }}, 5^{\text {th }}$, and $6^{\text {th }}$ cycles) velocity magnitude and $X$-vorticity contours of the computational data. $\left(R e=2605, k=0.56, \Pi_{1}=3.8 \times 10^{4}, \bar{\rho}=2233\right)$. 


\section{Instantaneous}

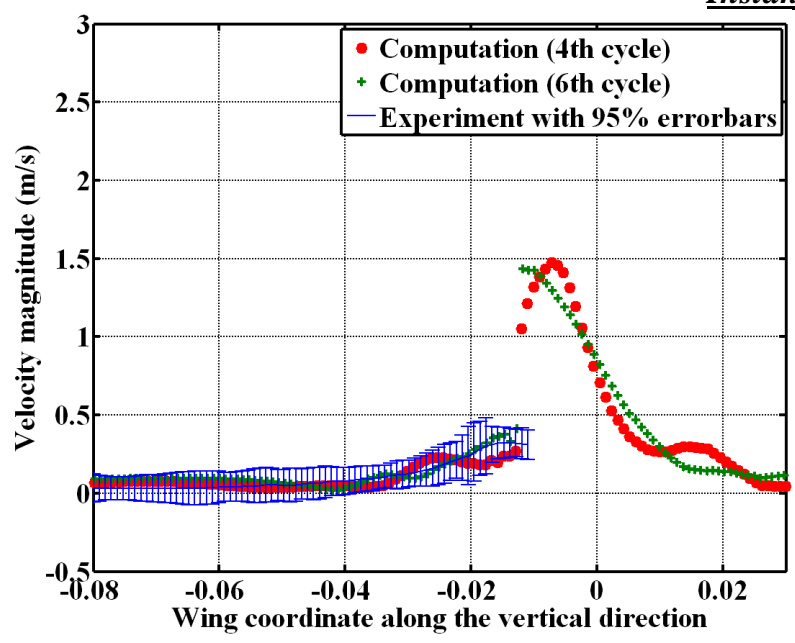

(A-1) - slice at the mid-span

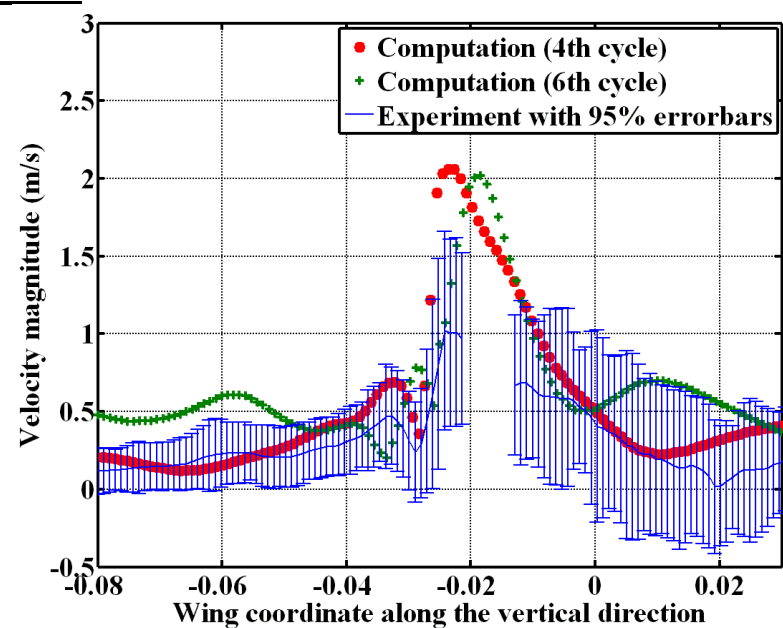

(B-1) - slice at the tip

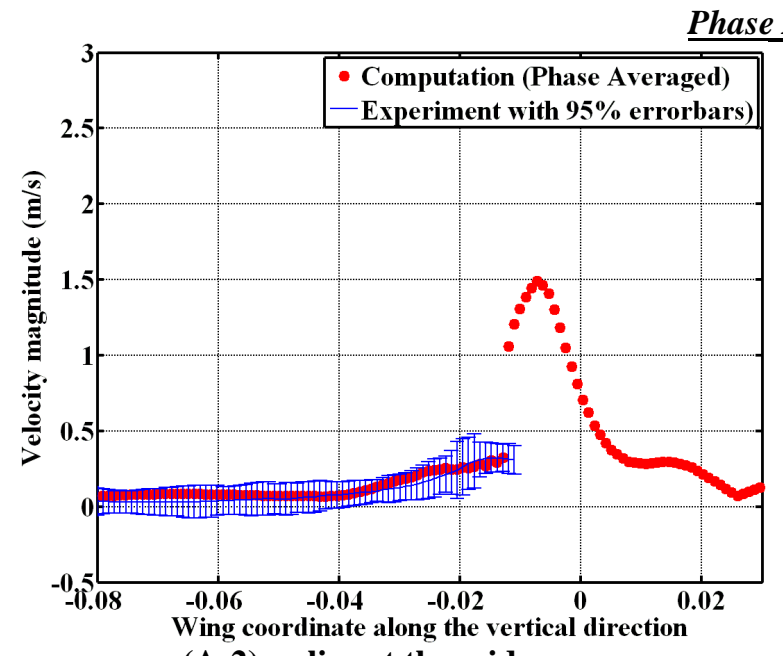

(A-2) - slice at the mid-span

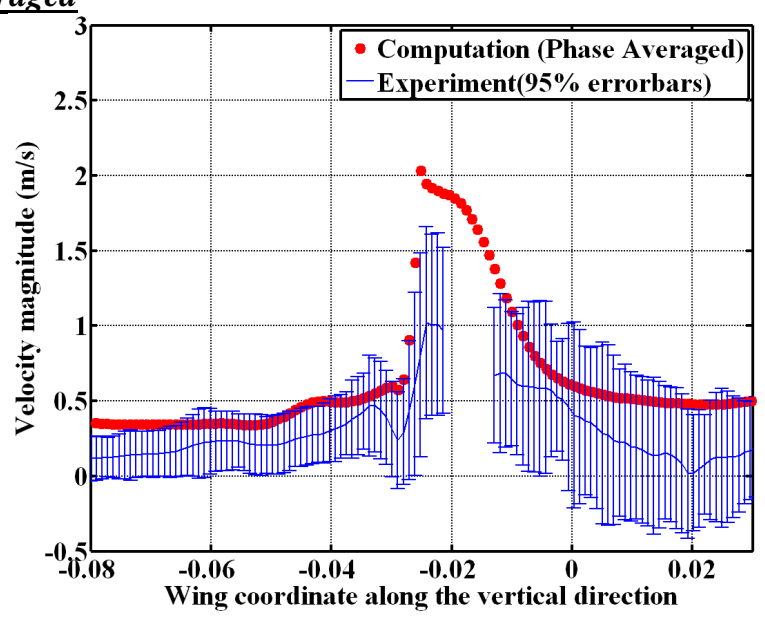

(B-2) - slice at the tip

Instantaneous

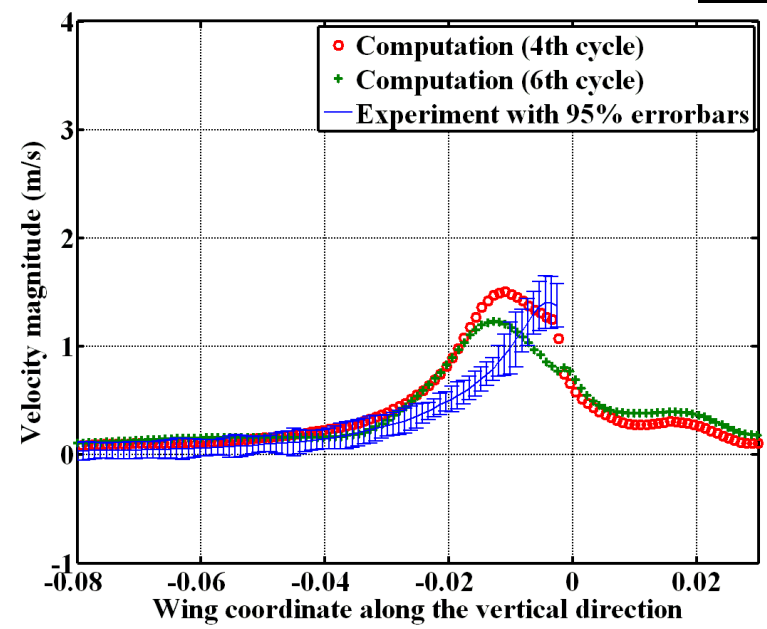

(C-1) - slice at the mid-span

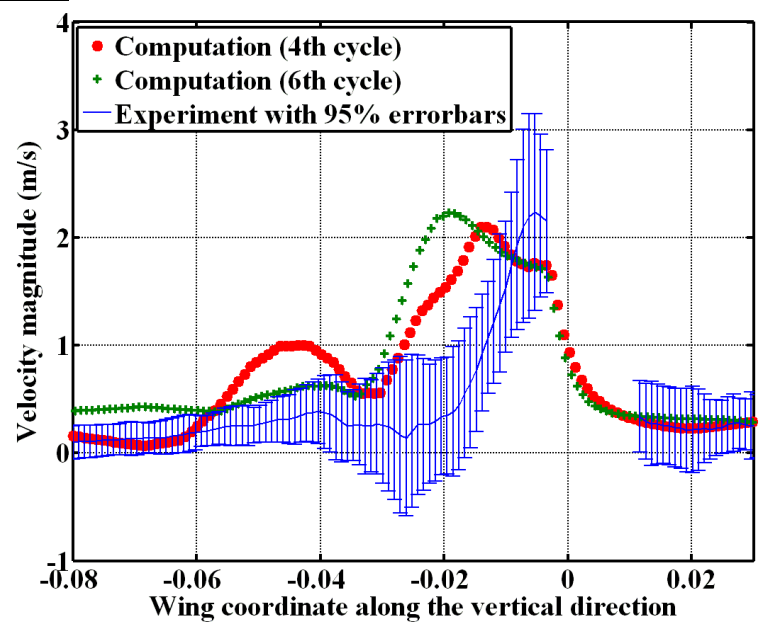

(D-1) - slice at the tip 


\section{Phase Averaged}

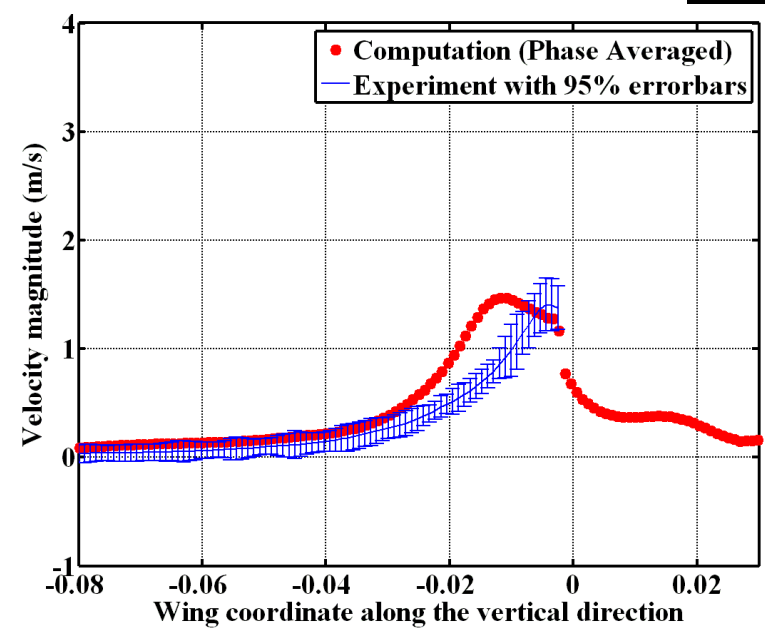

(C-2) - slice at the mid-span

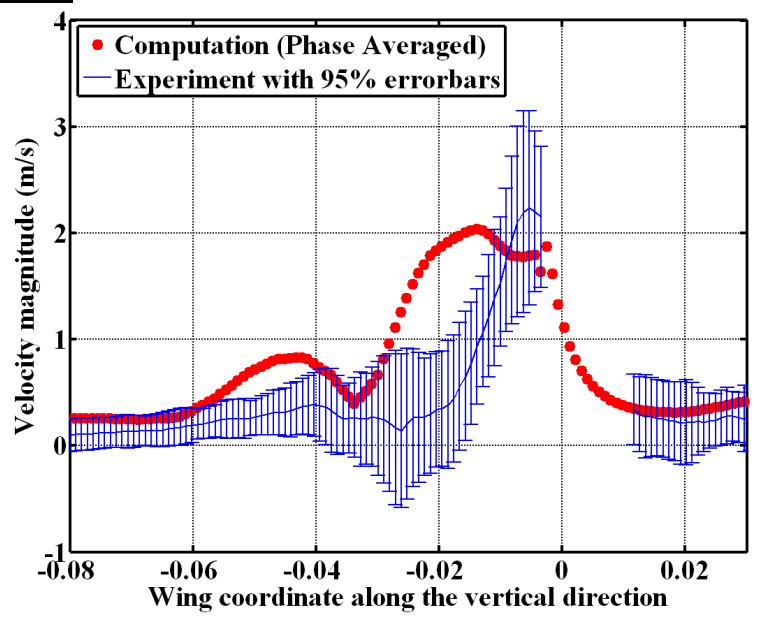

(D-2) - slice at the tip

Figure 5. Comparison of velocity magnitude between computation and experiment at two different time instants and for two different slices along the wing span: sub-plots A-1, B-1, C-1, and D-1 correspond to instantaneous velocity magnitude profiles and sub-plots A-2, B-2, C-2, and D-2 correspond to phase averaged velocity magnitude profiles. Sub-plots on the left column correspond to those at the mid-span slice of the wing and those on the right column correspond to a slice near the tip. The error-bars around the phase averaged flow indicate the interval within which $95 \%$ of the instantaneous values are expected to fall, assuming the distribution is Gaussian.

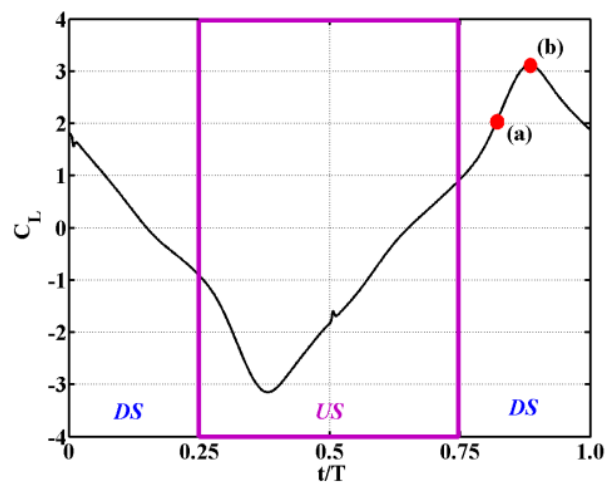

Figure 6. Lift coefficient on the wing as a function of normalized time (time is normalized with respect to a period of flap rotation), $D S$ - downstroke, $U S$ - upstroke

(i)

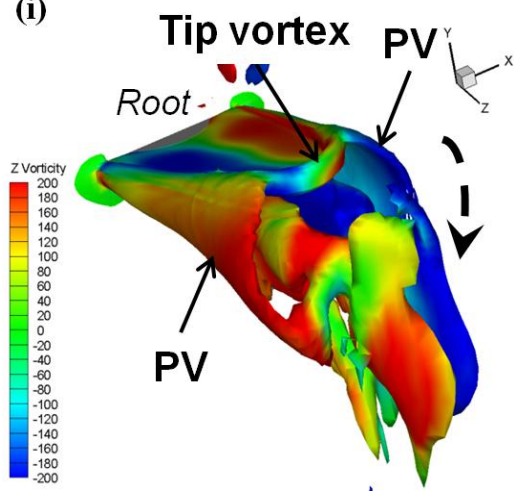

(ii)

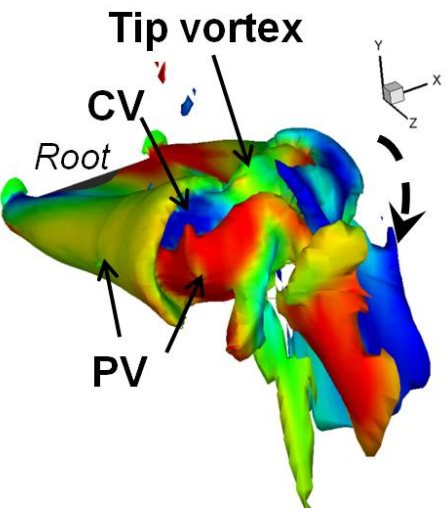




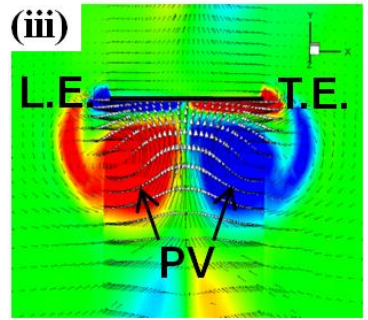

Mid span

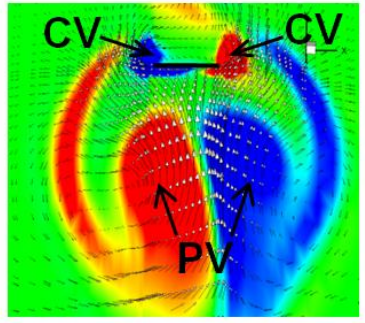

Near tip

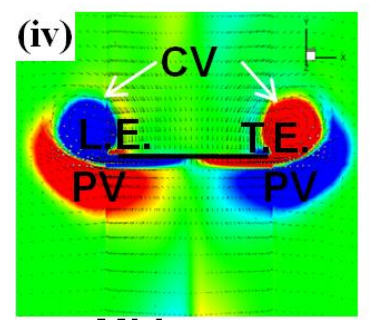

Mid span

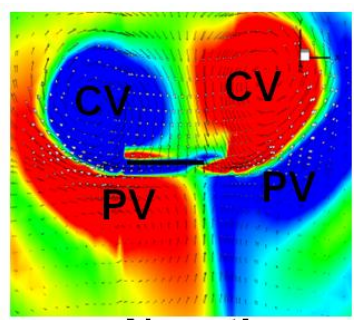

Near tip

Figure 7. Iso-contours of vorticity magnitude on the aluminum wing and $Z$-vorticity contours at two stations along the wing length at two different time instants: sub-plots (i) and (ii) correspond to the iso-contours of the vorticity magnitude (color indicates the magnitude of the $Z$-vorticity) for time instants (a) and (b) of Fig. 6 respectively. Sub-plots (iii) and (iv) correspond to the $Z$-vorticity contours at two stations along the wing length (as indicated) for time instants (a) and (b) of Fig. 6, respectively. (CV - Vortex generated in the current stroke, PV - vortex generated in the previous stroke, blue color indicates clockwise vorticity from the viewpoint of an observer looking into the plane of the plot).

Figure 8 shows the comparison of the normalized vertical displacement (with respect to the chord length at the wing root) at a point on the wing tip between the experiment and the computation. As shown in the figure, there is a decent overall agreement in both amplitude and phase between the computational response and the experimental data. The tip deformation obtained is only around $6 \%$ of wing chord which means that the selected wing configuration is not compliant enough to deform significantly. Future studies will be focused on more flexible cases.

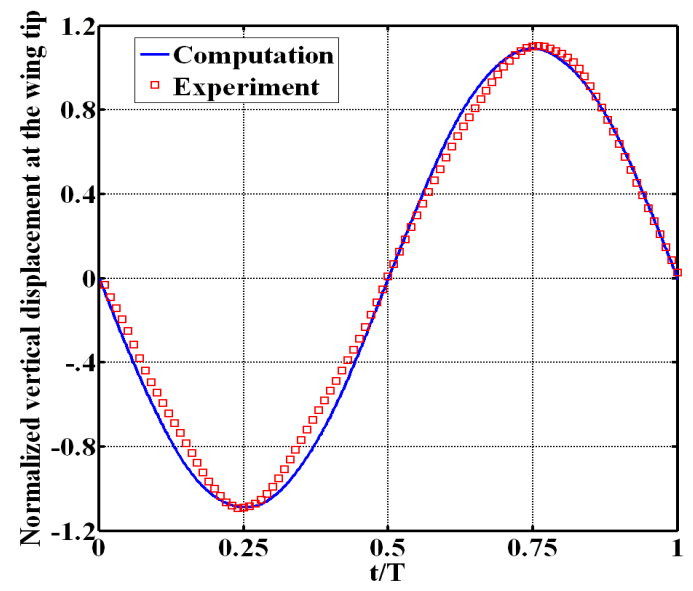

Figure 8. Aluminium wing response due to flapping excitation (normalized with respect to chord length at the root).

\section{Effect of Flexibility on Aerodynamics}

The experimental data is available only for the case of aluminium ( $E=70 \mathrm{GPa}$ and $\left.\Pi_{1}=38 \times 10^{3}\right)$, which is close to being rigid. Several variations in Young's modulus (effectively $\Pi_{1}$ ) are considered only in the computations to assess the impact of flexibility on aerodynamic force generation. A summary of the geometric and mechanical properties of the aluminum wing configuration studied in the computations is included in Table 4 . Table 5 provides information about the flow properties (dimensional). In Table 6, the key dimensionless parameters related to either the structure, the flow, or to both for the aluminum wing configuration are furnished. Figs. 9 and 10 show the lift and thrust coefficient response, respectively, for four cycles of computation for all cases of flexibility. It should be noted that for better clarity only data points skipping every ten time instants are considered in the plotting. As seen from the plots, the response corresponding to all wing configurations except the one with " $E=0.1 \mathrm{GPa}$ " seems to be close to periodic. 
Table 4. Geometric and mechanical properties associated with the test case.

\begin{tabular}{lcl}
\hline \hline Quantity & Symbol & Value \\
\hline \hline Semi-span at quarter chord & $R$ & $0.075 \mathrm{~m}$ \\
Chord length at wing root & $c_{\text {root }}$ & $0.025 \mathrm{~m}$ \\
Structural thickness & $h_{\mathrm{s}}$ & $0.4 \times 10^{-3} \mathrm{~m}$ \\
Poisson's ratio & $v$ & 0.3 \\
Material density & $\rho_{\mathrm{s}}$ & $2700 \mathrm{~kg} / \mathrm{m}^{3}$ \\
Young's modulus of material & $E$ & $0.1,10,30,50,70 \mathrm{GPa}$ \\
\hline \hline
\end{tabular}

Table 5. Flow properties associated with the test case.

\begin{tabular}{lcc}
\hline \hline Quantity & Symbol & Value \\
\hline \hline Reference flow velocity & $U_{\text {ref }}$ & $0.2618 \mathrm{~m} / \mathrm{s}$ (hover) \\
Air density & $\rho_{\mathrm{f}}$ & $1.209 \mathrm{~kg} / \mathrm{m}^{3}$ \\
\hline \hline
\end{tabular}

Table 6. Dimensionless parameters associated with the test case.

\begin{tabular}{lcl}
\hline Quantity & Symbol & Value \\
\hline \hline Mean chord-based Reynolds number & $R e$ & 620 \\
Reduced frequency & $k$ & 2.35 \\
Aspect ratio & $A_{\mathrm{R}}$ & 7.65 \\
Density ratio & $\bar{\rho}$ & 2233 \\
Scaling parameter - I & $\Pi_{1}$ & $937-\infty$ \\
\hline \hline
\end{tabular}

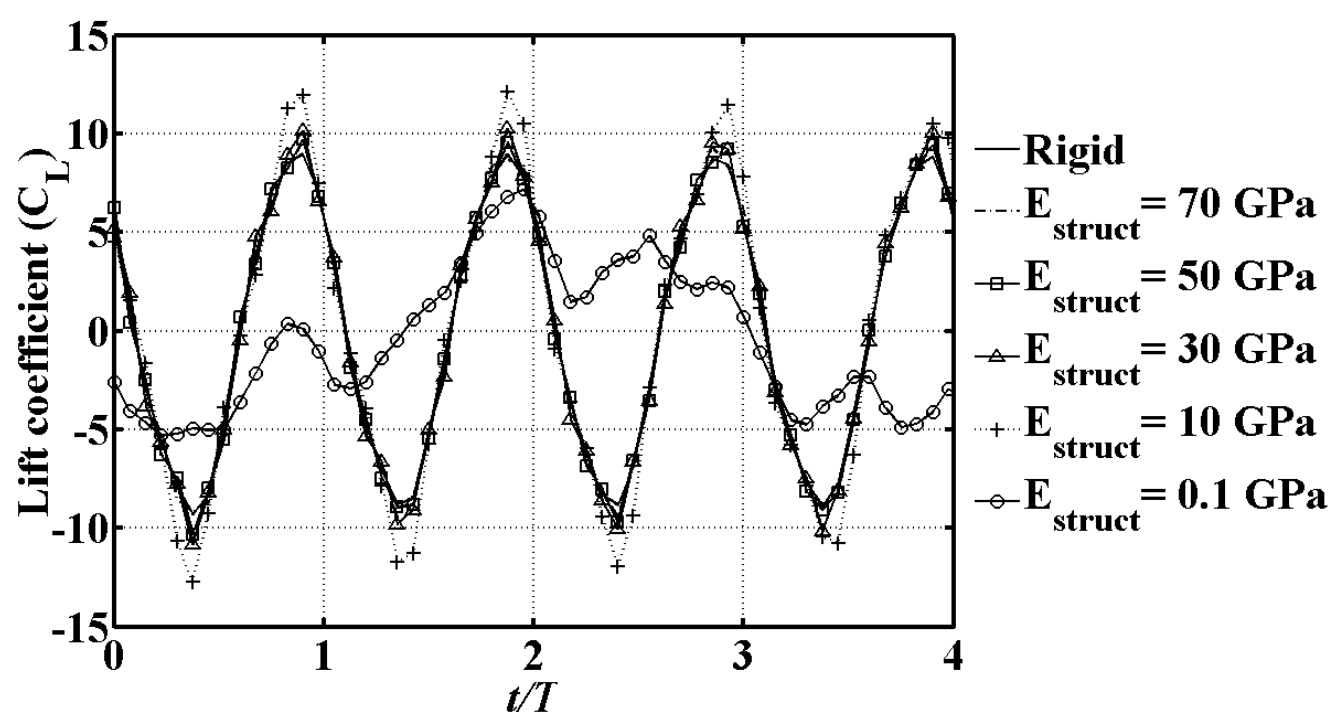

Figure 9. Lift coefficient response on the Zimmerman flapping wing with varying Young's modulus 


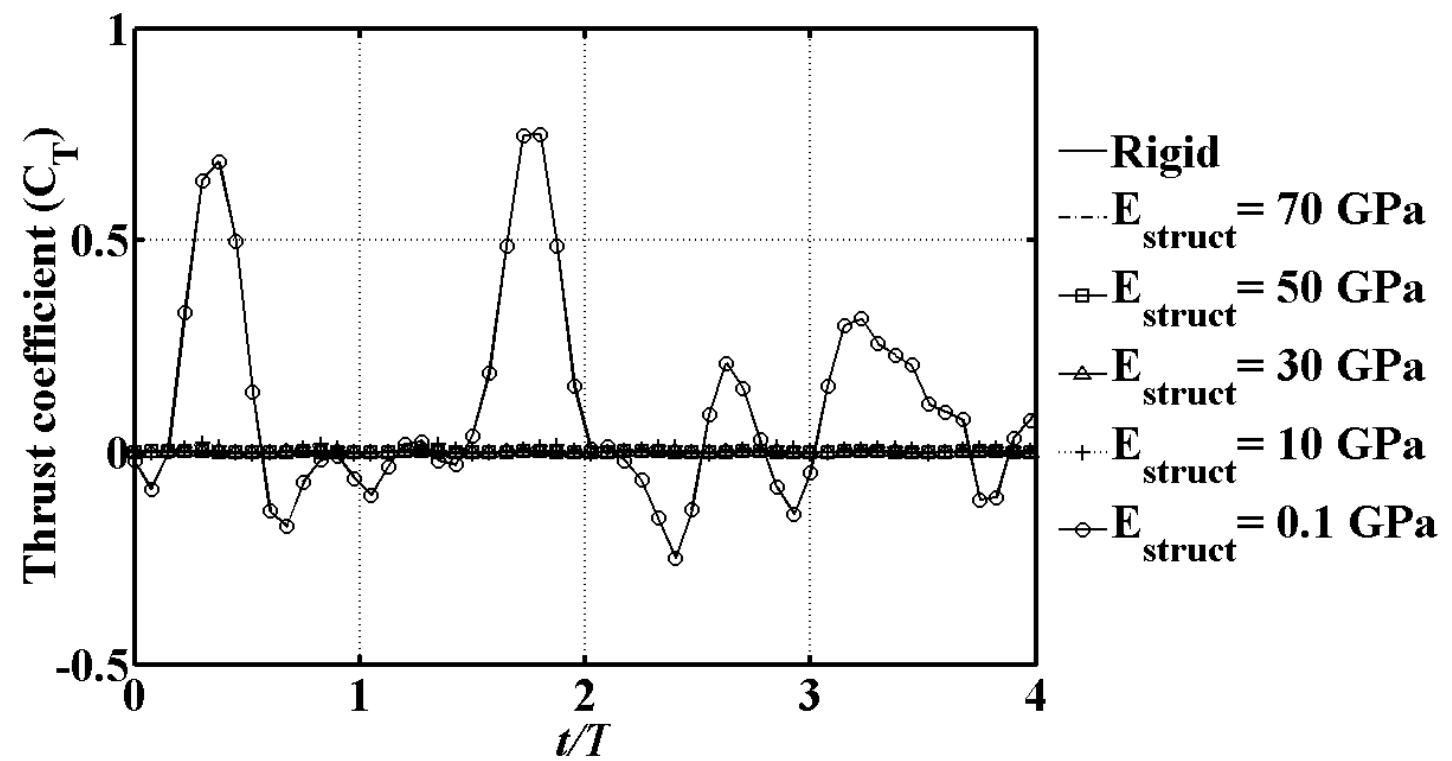

Figure 10. Thrust coefficient response on the Zimmerman flapping wing with varying Young's modulus

Figs. 11 and 12 give more insight into this by showing the mean lift and thrust coefficient, respectively, for all five cases. The standard deviation in the " $E=0.1 \mathrm{GPa"} \mathrm{case} \mathrm{is} \mathrm{so} \mathrm{large} \mathrm{for} \mathrm{the} \mathrm{mean} \mathrm{lift/thrust} \mathrm{coefficients} \mathrm{that} \mathrm{it} \mathrm{was}$ omitted from the plots. A reason for the aperiodicity in this case is the absence of enough aerodynamic damping which would reduce the structural dynamics general solution to the particular solution, which represents the steadystate vibration. Two key observations can be made from Figs. 11 and 12:

a. Within the range of flexibility considered, the mean thrust coefficient varies monotonically with increasing flexibility (due to the uncertainty in the mean values particularly for the " $E=10 \mathrm{GPa}$ " and " $E=0.1 \mathrm{GPa}$ " cases, no similar statement can be made for the mean lift coefficient).

b. While the increase in the thrust coefficient from "Rigid" to " $E=10 \mathrm{GPa}$ " is only minimum, it is more rapid from the latter to " $E=0.1 \mathrm{GPa"} \mathrm{case,} \mathrm{as} \mathrm{more} \mathrm{flexibility} \mathrm{is} \mathrm{added} \mathrm{to} \mathrm{the} \mathrm{system.}$

From this point, only the two extreme cases corresponding to "Rigid" and " $E=0.1 \mathrm{GPa"} \mathrm{are} \mathrm{considered} \mathrm{to} \mathrm{study} \mathrm{the}$ effects of flexibility on aerodynamic force generation. Figs. 13 and 14 show the lift and thrust coefficient response corresponding to these two cases for several cycles of computation. As seen from the plots, while the instantaneous lift coefficient on the "Rigid" wing configuration is much greater than that of " $E=0.1 \mathrm{GPa}$ " wing configuration, the trend is clearly opposite in the case of the thrust coefficient. Figs. 15 and 16 correspond to the pressure distribution contour plots on the top and bottom surfaces of the "Rigid" and " $E=0.1 \mathrm{GPa}$ " wings, respectively, at time instant D indicated in Figs. 13 and 14. It is seen from these plots that the top surface of the "Rigid" wing is dominated by suction more than that of the " $E=0.1 \mathrm{GPa"} \mathrm{wing} \mathrm{and} \mathrm{so,} \mathrm{it} \mathrm{was} \mathrm{found} \mathrm{that} \mathrm{the} \mathrm{total} \mathrm{resultant} \mathrm{force} \mathrm{of} \mathrm{the} \mathrm{"Rigid"}$ wing is more than that of the " $E=0.1 \mathrm{GPa"}$ at this time instant (and also at all others). Moreover, in the former, it is all in the form of lift whereas in the latter, due to the elastic twisting of the wing, there is also an effective horizontal force component (thrust). Fig. 17 illustrates this by showing the geometric twist angle at several stations along the span for both wing configurations. The geometric twist computed is the slope of the line joining the leading and trailing edge points corresponding to each station. In the figure, the data for the " $E=0.1 \mathrm{GPa"} \mathrm{case} \mathrm{is} \mathrm{shown} \mathrm{for}$ four different time instants A, B, C, and D indicated in Fig. 14. The data for the "Rigid wing" is shown only for one time instant since in that case the twist angle is zero at all time instants due to the absence of prescribed pitch. As seen in the figure, for the case of the " $E=0.1 \mathrm{GPa"} \mathrm{wing,} \mathrm{the} \mathrm{twist} \mathrm{angle} \mathrm{gradually} \mathrm{increases} \mathrm{from} \mathrm{root} \mathrm{to} \mathrm{the} \mathrm{tip} \mathrm{at}$ three time instants: A, C, and D. For time B, the behavior is non-monotonic. From the results presented above it seems that it is possible to tailor wing flexibility to alter the aerodynamics of a flapping wing. It will be interesting to conduct further studies by considering local variations in flexibility, which may better reflect the structural characteristics of the wings of an actual insect. 


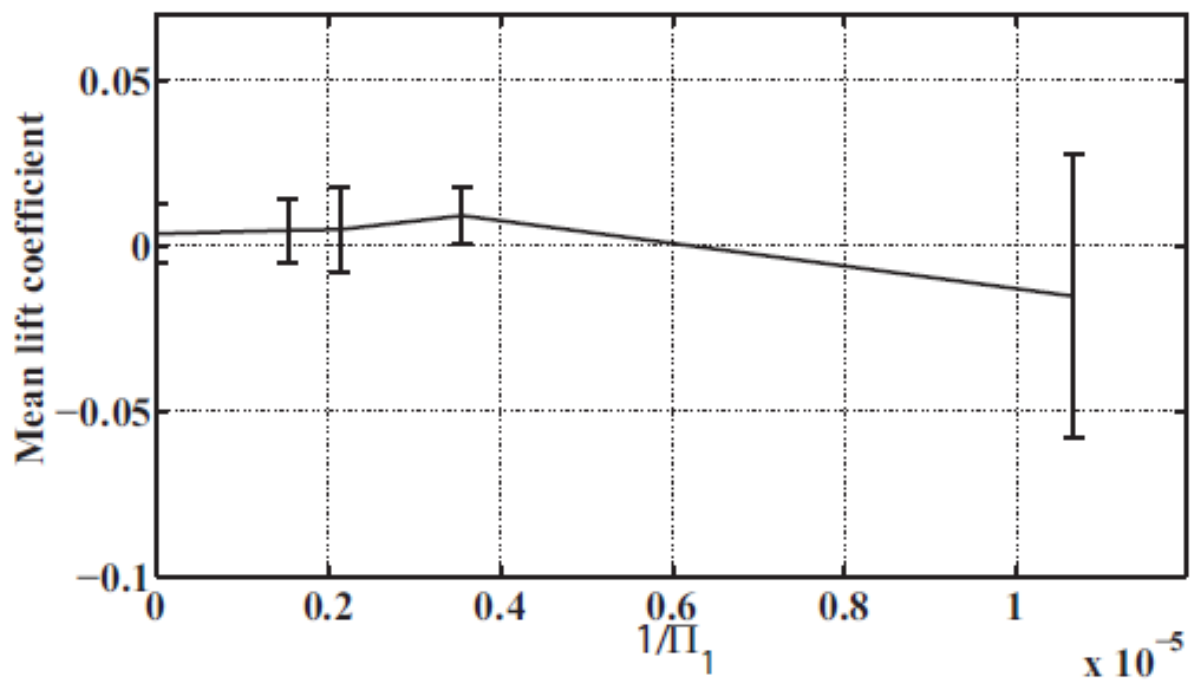

Figure 11. Mean and standard deviation of the mean lift coefficient of the hypothetical Zimmerman flapping wing configurations (standard deviation for $\Pi_{1}=938$ case is 2.08 )

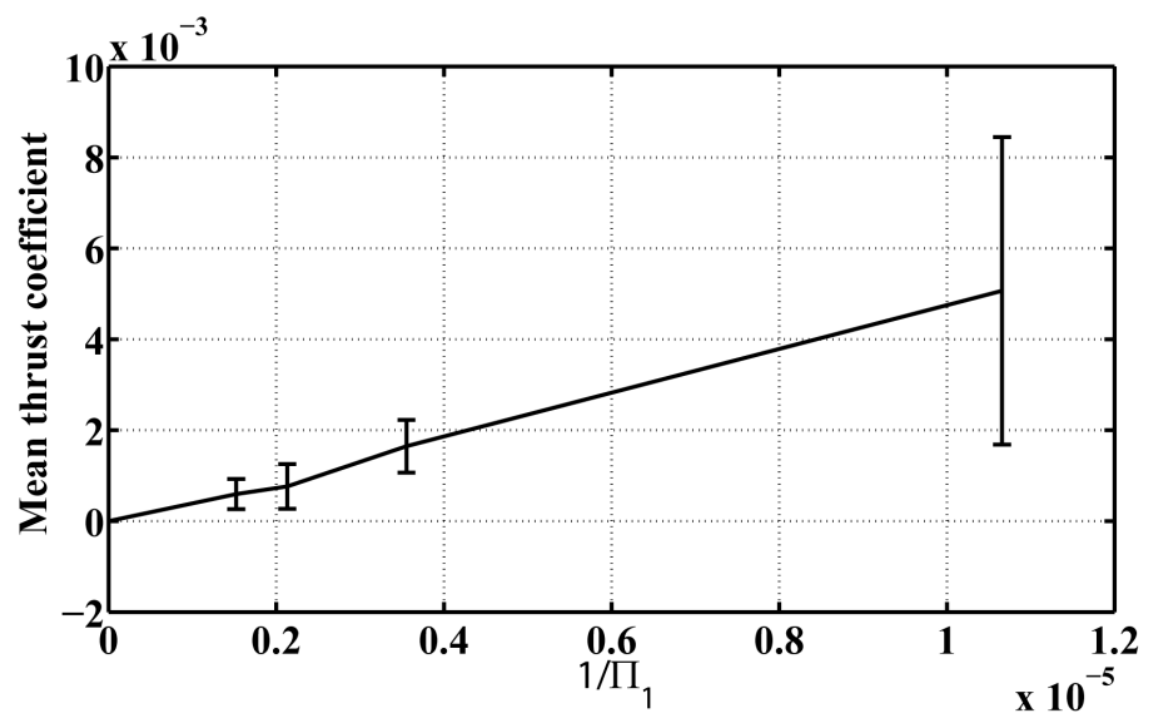

Figure 12. Mean and standard deviation of the mean thrust coefficient of the hypothetical Zimmerman flapping wing configurations (standard deviation for $\Pi_{1}=938$ case is 0.06 ) 


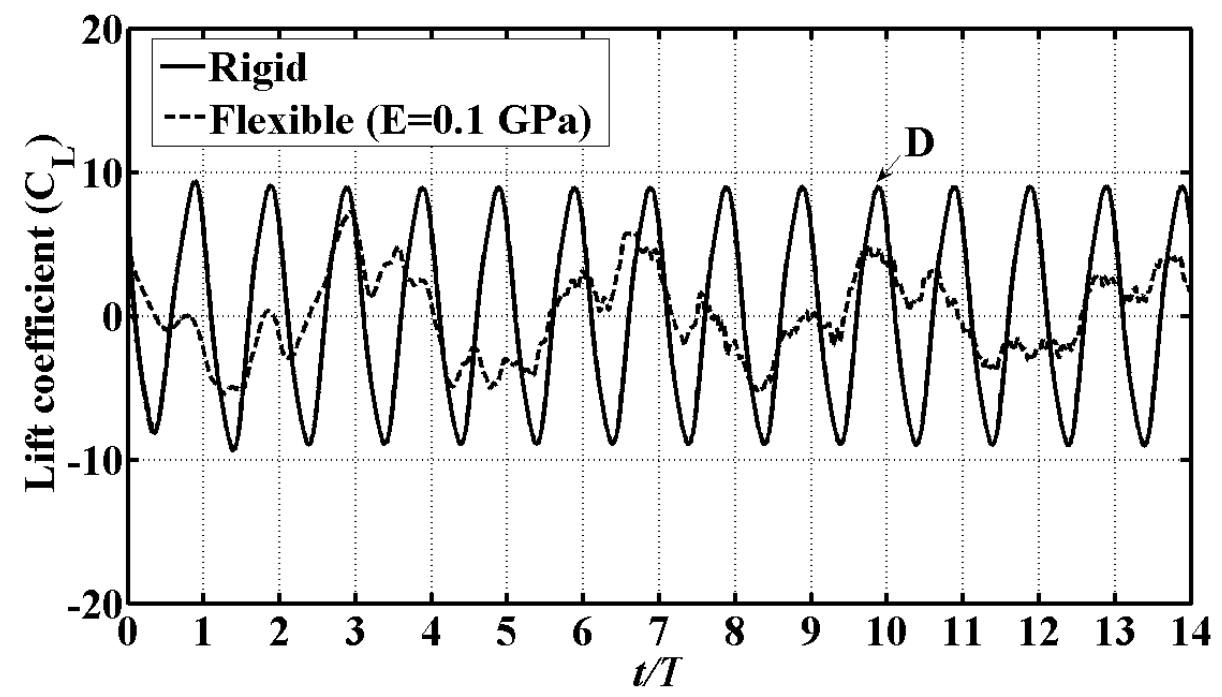

Figure 13. Lift coefficient response on the Zimmerman flapping wing for the "Rigid" and " $E=0.1 \mathrm{GPa}$ " cases.

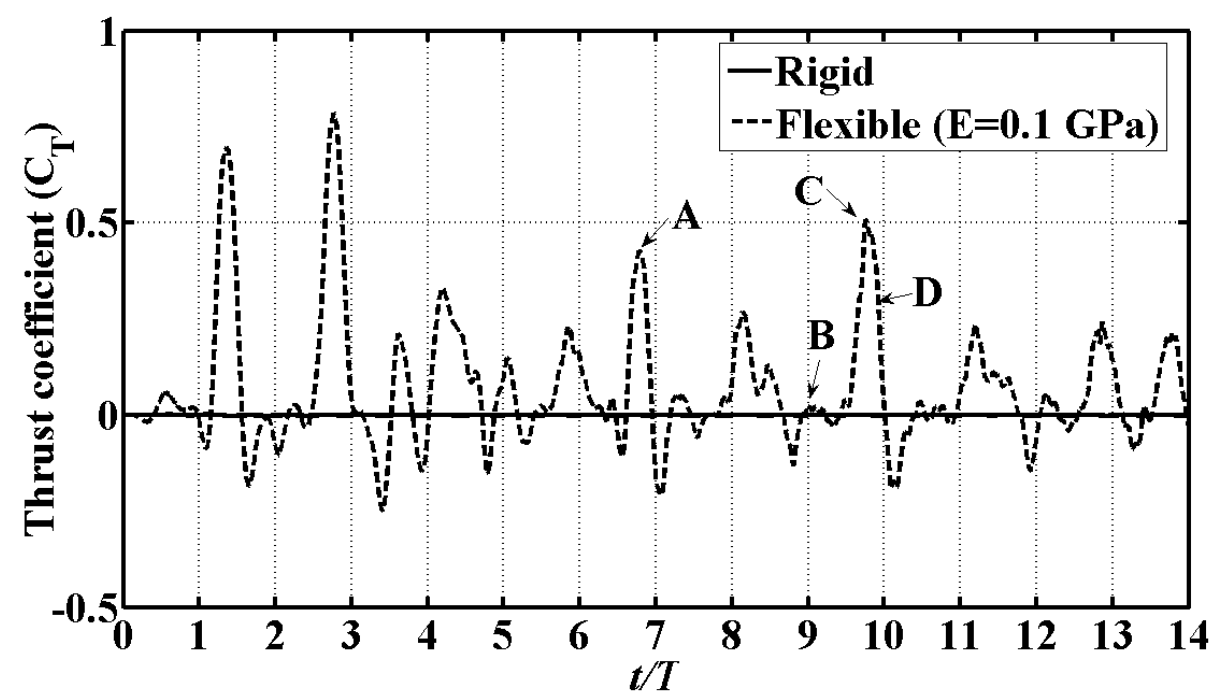

Figure 14. Thrust coefficient response on the Zimmerman flapping wing for the "Rigid" and " $E=0.1 \mathrm{GPa}$ " cases. 

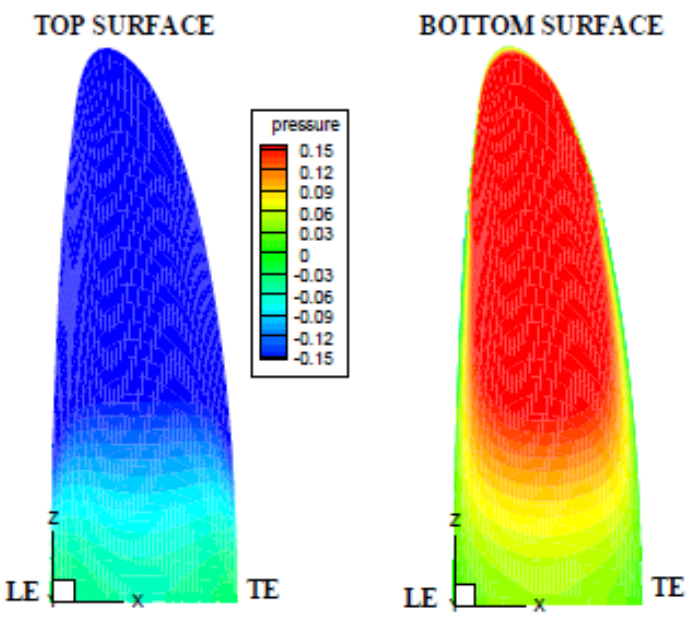

Figure 15. Pressure distribution on the top and bottom surfaces of the "Rigid" Zimmerman flapping wing at time instant $D$ indicated in Fig. 13
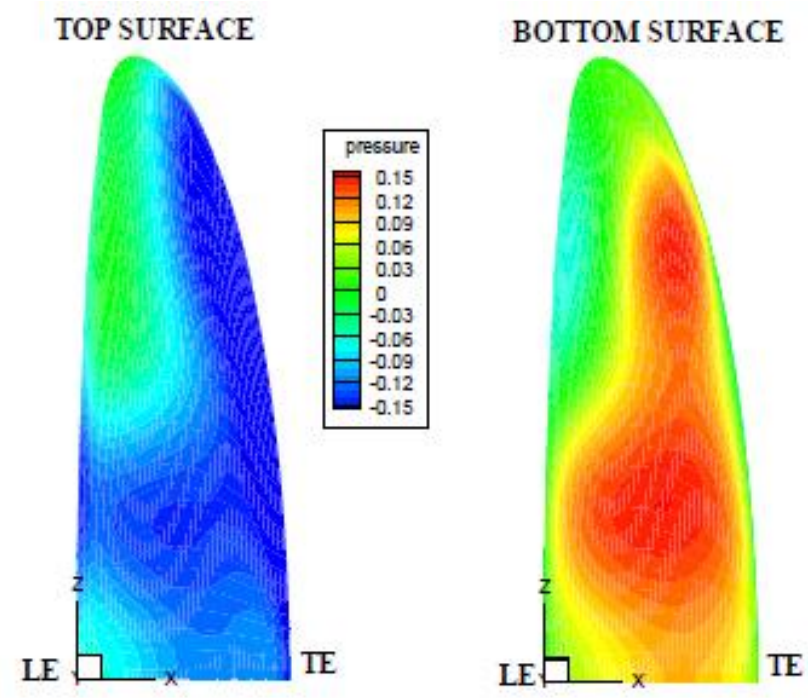

Figure 16. Pressure distribution on the top and bottom surfaces of the " $E=0.1 \mathrm{GPa}$ " Zimmerman flapping wing at time instant $D$ indicated in Fig. 13 


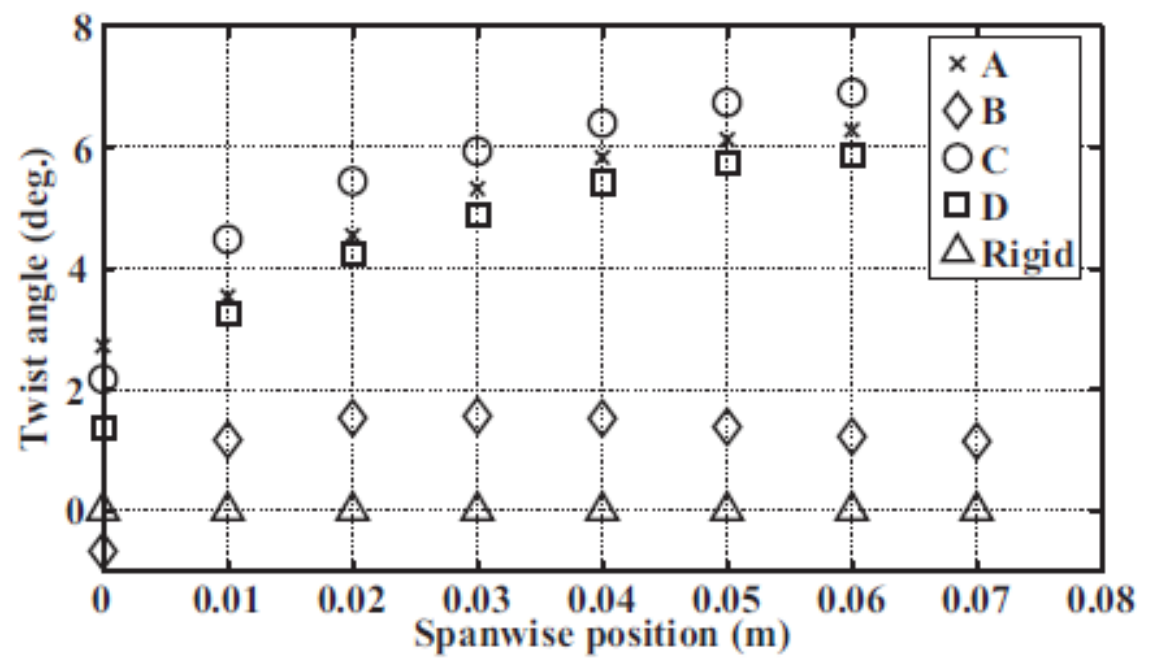

Figure 17. Geometric twist angle along the span for the "Rigid" and " $E=0.1$ GPa" Zimmerman flapping wing configurations at time instants A, B, C, and D indicated in Fig. 14.

\section{Concluding Remarks}

This paper has presented an integrated approach involving both experimental and computational methods having a potential to examine flexible flapping wing configurations. An aluminum wing is prescribed with single degree-offreedom flap rotation at $10 \mathrm{~Hz}$ frequency and $\pm 21^{\circ}$ amplitude and both flow velocities and deformations are measured in the experiment using digital image correlation and digital particle image velocimetry techniques respectively. Specific comparison of flow velocities and wing deformation between the computational and the experimental data showed a reasonable agreement. Flow structures including three-dimensional vortex generation are observed. The counter-rotating vortices at the leading and the trailing edge interact with the tip vortex during the wing motion. The vortices generated during a previous stroke are captured by the wing and interact with the vortices generated during the present stroke. Detailed velocity profiles and force time histories are documented. Studies on additional flexible flapping wing configurations showed that aerodynamic force generation could be enhanced due to wing flexibility. In particular, in the most flexible flapping wing case, the elastic twisting of the wing was shown to produce substantially larger mean and instantaneous thrust due to shape deformation-induced changes in effective angle of attack. Overall, it seems that it is possible to tailor wing flexibility to alter the aerodynamics of a flapping wing. It will be interesting to conduct further studies by considering local variations in flexibility, which may better reflect the structural characteristics of the wings of an actual insect.

\section{Acknowledgments}

The work supported here has been supported in part by the Air Force Office of Scientific Research's Multidisciplinary University Research Initiative (MURI) grant and by the Michigan/AFRL (Air Force Research Laboratory)/Boeing Collaborative Center in Aeronautical Sciences.

\section{References}

${ }^{1}$ Shyy, W., Lian, Y., Tang, J., Viieru, D., and Liu, H., Aerodynamics of Low Reynolds Number Flyers, Cambridge University Press, New York, 2008.

${ }^{2}$ Shyy W, Berg M, Ljungqvist D. Flapping and flexible wings for biological and micro air vehicles. Prog Aerosp Sci 1999; 35 (5): 455-505.

${ }^{3}$ Muller TJ. Fixed and flapping wing aerodynamics for micro air vehicle applications. AIAA Prog Astronaut Aeronaut $2002 ; 195$.

${ }^{4}$ Shyy W, Ifju P, Viieru D. Membrane wing-based micro air vehicles. Appl Mech Rev 2005; 58: 283-301.

${ }^{5}$ Pines DJ, Bohorquez F. Challenges facing future micro-air-vehicle development. J Aircraft 2006; 43(2): $290-305$. 
${ }^{6}$ Platzer M, Jones K, Young J, Lai J. Flapping wing aerodynamics: Progress and challenges. AIAA J 2008; 46(9): $2136-2149$.

${ }^{7}$ Lian Y, Shyy W, Viieru D, Zhang B. Membrane wing aerodynamics for micro air vehicles. Prog Aerosp Sci 2003; 39 (6-7): 425-465.

${ }^{8}$ Stanford BK, Ifju P, Albertani R, Shyy W. Fixed membrane wings for micro air vehicles: Experimental characterization, numerical modeling, and tailoring. Prog Aerosp Sci 2008; 44 (4): 258-294.

${ }^{9}$ Dalton S. The miracle of flight. London: Merrell, 2006.

${ }^{10}$ Daniel TL, Combes SA. Flexible wings and fins: bending by inertial or fluid-dynamic forces? Integr Comp Biol 2002; 42 : 1044-1049.

${ }^{11}$ Combes SA. Wing flexibility and design for animal flight. Ph.D Diss. 2002 University of Washington.

${ }^{12}$ Combes SA, Daniel TL. Into thin air: contributions of aerodynamic and inertial-elastic forces to wing bending in the hawkmoth Manduca sexta. J Exp Biol 2003; 23: 2999-3006.

${ }^{13}$ Mountcastle AM, Daniel TL. Aerodynamic and functional consequences of wing compliance. Exp Fluids 2009; 46: 873-882.

${ }^{14} \mathrm{Zhu}$ Q. Numerical simulation of a flapping foil with chordwise or spanwise flexibility. AIAA J 2007; 45(10): $2448-2457$.

${ }^{15}$ Heathcote S, Gursul I. Flexible flapping airfoil propulsion at low Reynolds numbers. AIAA J 2007; 45 (5): $1066-1079$.

${ }^{16}$ Yamamoto I, Terada Y, Nagamatu T, Imaizumi Y. Propulsion system with flexible/rigid oscillating fin. IEEE J Oceanic Eng 1995; 20 (1): 23-30.

${ }^{17}$ Prempraneerach P, Hover FS, Triantafyllou MS. The effect of chordwise flexibility on the thrust and efficiency of a flapping foil. Proceedings of $13^{\text {th }}$ International Symposium Unmanned Untethered Submersible Technology 2003. p. 1-10.

${ }^{18}$ Tang J, Viieru D, Shyy W. A study of aerodynamics of low Reynolds number flexible airfoils. Proceedings of the AIAA $37^{\text {th }}$ Fluid Dynamics Conference and Exhibit AIAA 2007-4212. 2007. p. 1-17.

${ }^{19}$ Chandar DDJ, Damodaran M. Computational fluid-structure interaction of a flapping wing in free flight using overlapping grids. Proceedings of $27^{\text {th }}$ AIAA Applied Aerodynamics Conference AIAA 2009-3849. 2009. p. 1-18.

${ }^{20}$ Pederzani J, Haj-Hariri H. Numerical analysis of heaving flexible airfoils in a viscous flow. AIAA J 2006; 44 (11): $2773-2779$.

${ }^{21}$ Chaithanya MR, Venkatraman K. Hydrodynamic propulsion of a flexible foil. Proceedings of the $12^{\text {th }}$ Asian Congress of Fluid Mechanics. 2008. p. 1-5.

${ }^{22}$ Chaithanya MR, Venkatraman K. Hydrodynamic propulsion of a flexible foil undergoing pitching motion. Proceedings of the $10^{\text {th }}$ Annual CFD Symposium. 2008. p. 1-5.

${ }^{23}$ Gopalakrishnan P. Unsteady Aerodynamic and Aeroelastic Analysis of Flapping Flight. PhD Dissertation, Department of Mechanical Engineering, Virginia Polytechnic Institute and State University, Blacksburg, Virginia, 2008.

${ }^{24}$ Miao J-M, Ho M-H. Effect of flexure on aerodynamic propulsive efficiency of flapping flexible airfoil. J Fluid Struct 2006; 22 : 401-409.

${ }^{25}$ Toomey J, Eldredge JD. Numerical and experimental study of the fluid dynamics of a flapping wing with low order flexibility. Phys Fluids 2008; 20 (073603): 1-10.

${ }^{26}$ Vanella M, Fitzgerald T, Preidikman S, Balaras E, Balachandran B. Influence of flexibility on the aerodynamic performance of a hovering wing. J Exp Biol 2009; 212: 95-105.

${ }^{27}$ Heathcote S, Martin D, Gursul I. Flexible flapping airfoil propulsion at zero freestream velocity. AIAA J $2004 ; 42$ (11): $2196-$ 2204.

${ }^{28}$ Michelin S, Smith SGL. Resonance and propulsion performance of a heaving flexible wing. Phys Fluids 2009; 21: 071902-1071902-15.

${ }^{29}$ Liu P, Bose N. Propulsive performance from oscillating Propulsors with spanwise flexibility. P Roy Soc A-Math Phy $1997 ; 453$ (1963): 1763-1770.

${ }^{30}$ Heathcote S, Wang Z, Gursul I. Effect of spanwise flexibility on flapping wing propulsion. J Fluid Struct 2008; 24: $183-199$.

${ }^{31}$ Hamamoto M, Ohta Y, Hara K, Hisada T. Application of fluid-structure interaction analysis to flapping flight of insects with deformable wings. Adv Robotics 2007; 21 (1-2): 1-21.

${ }^{32}$ Singh B, Chopra I. Insect-based hover-capable flapping wings for micro air vehicles: Experiments and analysis. AIAA J 2008; 46 (9): 2115-2135.

${ }^{33}$ Young J, Walker SM, Bomphrey RJ, Taylor GK, Thomas ALR. Details of insect wing design and deformation enhance aerodynamic function and flight efficiency. Science 2009; 325: 1549-1552.

${ }^{34}$ Walker SM, Thomas ALR, Taylor GK. Deformable wing kinematics in the desert locust: how and why do camber, twist and topography vary through the stroke? J R Soc Interface 2008; 6 (38): 735-747.

${ }^{35}$ Agrawal A, Agrawal SK. Design of bio-inspired flexible wings for flapping-wing micro-sized air vehicle applications. Adv Robotics 2009; 23(7-8): 979-1002.

${ }^{36} \mathrm{Wu}$ P, Ifju P, Standford BK, Sällström E, Ukeiley L, Love R, Lind R. A multidisplinary experimental study of flapping wing aeroelasticity in thrust production. Proceedings of $50^{\text {th }}$ AIAA/ASME/ASCE/AHS/ASC Structures, Structural Dynamics, and Materials Conference AIAA 2009-2413. 2009. p. 1-19.

${ }^{37}$ Sällström E, Ukeiley L, Pin W, Ifju P. Three-dimensional averaged flow and wing deformation around flexible flapping wings. Proceedings of $39^{\text {th }}$ AIAA Fluid Dynamics Conference AIAA 2009-3813. 2009. p. 1-13.

${ }^{38}$ Chimakurthi SK, Tang J, Palacios R, Cesnik CES, Shyy W. Computational aeroelasticity framework for analyzing flapping wing micro air vehicles. AIAA J 2009; 47(8): 1865-1878.

${ }^{39}$ Aono H, Chimakurthi SK, Cesnik CES, Liu H, Shyy W. Computational modeling of spanwise flexibility effects on flapping wing aerodynamics. Proceedings of $47^{\text {th }}$ AIAA Aerospace Sciences Meeting Including The New Horizons Forum and Aerospace Exposition AIAA 2009-1270. 2009. p. 1-18. 
${ }^{40}$ Chimakurthi, SK, Stanford, BK, Cesnik, CES, Shyy, W, Flapping wing CFD/CSD aeroelastic formulation based on a corotational shell finite element. 50th AIAA/ASME/ASCE Structural Dynamics and Materials Conference, Palm Springs, California, 3-7 May 2009.

${ }^{41}$ Battini, J, Pacoste, C. On the choice of local element frame for co-rotational triangular shell elements. Commun Numer Meth En 2004; 20: 819-825.

${ }^{42}$ Battini, JM. A modified co-rotational framework for triangular shell elements. Comput Method Appl M 2007; 196 : $1905-1914$.

${ }^{43}$ Crisfield, M, Galvanetto, U, Jelenic, G. Dynamics of 3-D co-rotational beams. Comput Mech 1997; 20: 507-519.

${ }^{44}$ Devloo, P, Geradin, M, Fleury, R. A co-rotational formulation for the simulation of flexible mechanisms. Multibody Syst Dyn 2000; 4: 267-295.

${ }^{45}$ Elkaranshawy, HA, Dokainish, MA. Co-rotational finite element analysis of planar flexible multibody systems. Computer Struct 1995; 54: 881-890.

${ }^{46}$ Thomas, PD, Lombard, K. Geometric conservation law and its application to flow computations on moving grids. AIAA J 1979; 17(10): 1030-1037.

${ }^{47}$ Shyy, W, Udaykumar, HS, Rao, MM, Smith, RW. Computational fluid dynamics with moving boundaries, Dover, New York, 2007. 